\title{
Extremely anisotropic van der Waals thermal conductors
}

https://doi.org/10.1038/s41586-021-03867-8

Received: 5 February 2021

Accepted: 29 July 2021

Published online: 29 September 2021

\section{Open access}

Check for updates

\author{
Shi En Kim1, Fauzia Mujid², Akash Rai ${ }^{3}$, Fredrik Eriksson ${ }^{4}$, Joonki Suh ${ }^{2,5}$, Preeti Poddar², \\ Ariana Ray ${ }^{6}$, Chibeom Park ${ }^{1,5}$, Erik Fransson ${ }^{4}$, Yu Zhong ${ }^{2}$, David A. Muller ${ }^{6}$, Paul Erhart ${ }^{4 凶}$, \\ David G. Cahill ${ }^{3 凶}$ \& Jiwoong Park ${ }^{1,2,5 \bowtie}$
}

The densification of integrated circuits requires thermal management strategies and high thermal conductivity materials ${ }^{1-3}$. Recent innovations include the development of materials with thermal conduction anisotropy, which can remove hotspots along the fast-axis direction and provide thermal insulation along the slow axis ${ }^{4,5}$. However, most artificially engineered thermal conductors have anisotropy ratios much smaller than those seen in naturally anisotropic materials. Here we report extremely anisotropic thermal conductors based on large-area van der Waals thin films with random interlayer rotations, which produce a room-temperature thermal anisotropy ratio close to 900 in $\mathrm{MoS}_{2}$, one of the highest ever reported. This is enabled by the interlayer rotations that impede the through-plane thermal transport, while the long-range intralayer crystallinity maintains high in-plane thermal conductivity. We measure ultralow thermal conductivities in the through-plane direction for $\mathrm{MoS}_{2}$ $\left(57 \pm 3 \mathrm{~mW} \mathrm{~m}^{-1} \mathrm{~K}^{-1}\right)$ and $\mathrm{WS}_{2}\left(41 \pm 3 \mathrm{~mW} \mathrm{~m}^{-1} \mathrm{~K}^{-1}\right)$ films, and we quantitatively explain these values using molecular dynamics simulations that reveal one-dimensional glass-like thermal transport. Conversely, the in-plane thermal conductivity in these $\mathrm{MoS}_{2}$ films is close to the single-crystal value. Covering nanofabricated gold electrodes with our anisotropic films prevents overheating of the electrodes and blocks heat from reaching the device surface. Our work establishes interlayer rotation in crystalline layered materials as a new degree of freedom for engineering-directed heat transport in solid-state systems.
Anisotropic thermal conductors, in which heat flows faster in one direction than in another, can be characterized by the thermal conductivity anisotropy ratio $\rho\left(=\kappa_{\mathrm{f}} / \kappa_{\mathrm{s}}\right)$ between the thermal conductivities along the fast axis $\left(\kappa_{\mathrm{f}}\right)$ and the slow axis $\left(\kappa_{\mathrm{s}}\right)$. One common way to engineer $\rho$ in fully dense solids is via nanostructuring ${ }^{6}$, such as fabricating inorganic superlattices ${ }^{7-11}$ or designing symmetry-breaking crystal architectures in a single material ${ }^{12}$. However, such engineered materials have relatively small $\rho$ values of less than 20 at room temperature. Conversely, some natural crystalline materials have an intrinsically large $\rho$ (for example, graphite ${ }^{1}$ and hexagonal boron nitride $(\mathrm{hBN})^{13}$, with $\rho \approx 340$ and 90 respectively), but they are often difficult to process scalably for thin film integration. Some of these films may also lack the electrical or optical properties necessary for functional device applications.

To design materials with higher $\rho$ that are also suitable for real-world applications, an approach needs to be developed to include three key features: (1) a candidate material with intrinsically high $\kappa_{\mathrm{f}}$, usually one with efficient phonon-mediated thermal transport; (2) a method to substantially reduce $\kappa_{\mathrm{s}}$ without affecting $\kappa_{\mathrm{f}}$; and (3) facile, scalable production and integration of such a material with precise control of the material dimensions (for example, film thickness). Layered van der Waals (vdW) materials such as graphite and transition metal dichalcogenides
(TMDs) provide an ideal material platform for designing such high- $\rho$ materials. They generally have excellent intrinsic in-plane thermal conductivities $\left(\boldsymbol{\kappa}_{\|}\right)$in single-crystalline form. Previous studies have also measured record-low thermal conductivities in nanocrystalline vdW films (for example, $\left.\mathrm{WSe}_{2}\right)^{14-17}$ and heterostructures ${ }^{18}$. One currently missing capability, however, is an approach for significantly decreasing the out-of-plane thermal conductivity $\left(\kappa_{\perp}\right)$ while maintaining high $\kappa_{\|}$.

\section{TMD films with interlayer rotations}

Here we show that such capability is provided by interlayer rotations, as illustrated in Fig. 1a. Interlayer rotation breaks the through-plane translational symmetry at the atomic scale while retaining in-plane long-range crystallinity in each monolayer, thereby providing an effective means for suppressing only $\kappa_{\perp}$. For this, we produce large-area TMD films without interlayer registry (referred to here as $r$-TMD), which possess long-range crystallinity in-plane and relative lattice rotations at every interlayer interface (Fig. 1b). The films are produced in large-scale using two steps: wafer-scale growth of continuous TMD monolayers (polycrystalline; domain size $D$ ) and layer-by-layer stacking in vacuum using previously reported methods ${ }^{19,20}$ (see Methods).

${ }^{1}$ Pritzker School of Molecular Engineering, University of Chicago, Chicago, IL, USA. ${ }^{2}$ Department of Chemistry, University of Chicago, Chicago, IL, USA. ${ }^{3}$ Department of Materials Science and

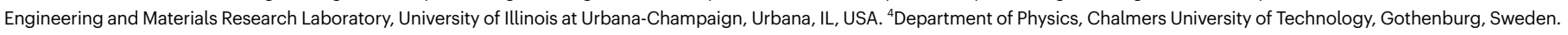

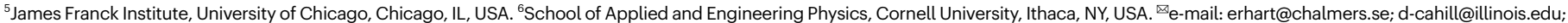
jwpark@uchicago.edu 

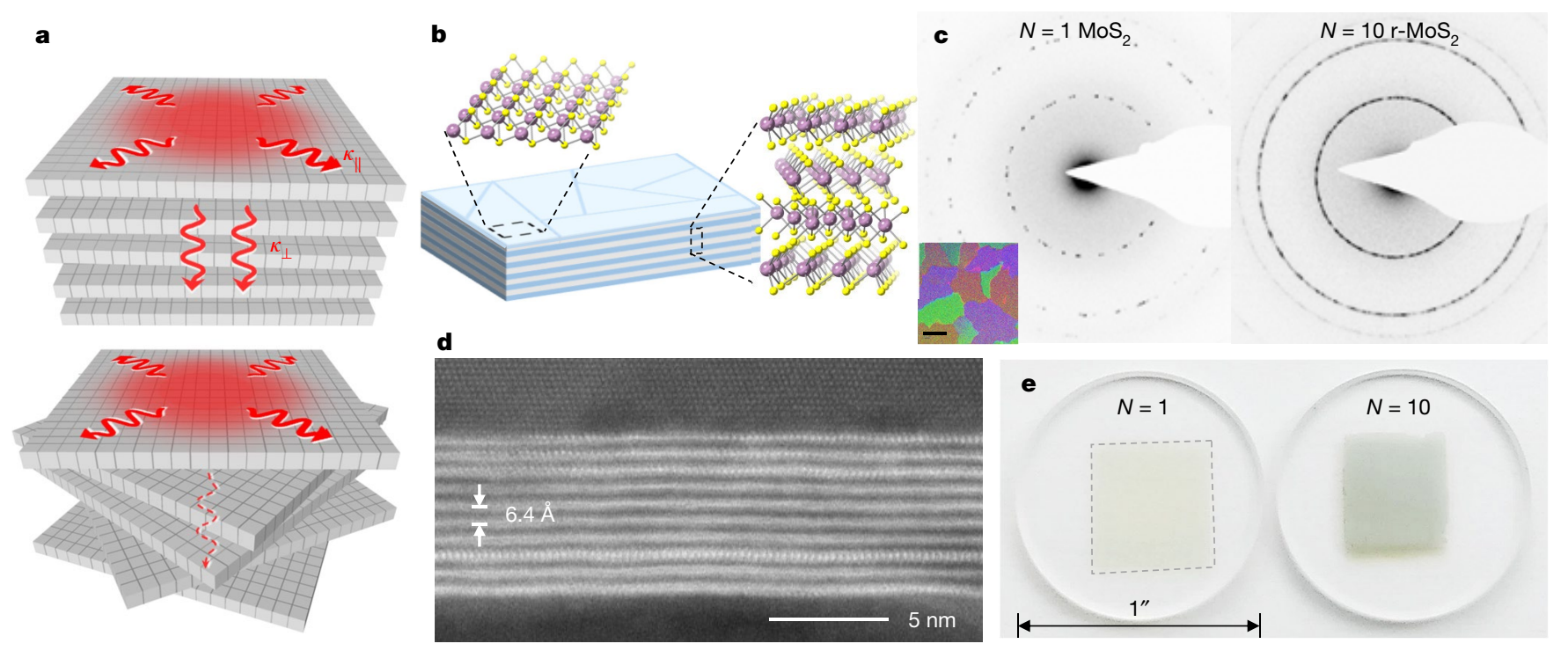

Fig. 1|Structure of r-TMD films.a, Conceptual strategy for engineering thermal anisotropy in a single material system, using random interlayer rotation in polycrystalline vdW layered materials. b, Schematic of an r-MoS film with random crystalline orientation. c, Greyscale-inverted TEM electron diffraction patterns probed from a $500 \mathrm{~nm} \times 500 \mathrm{~nm}$ area of a monolayer and an $N=10 \mathrm{r}-\mathrm{MoS}_{2}$ film. Inset: darkfield TEM image of a monolayer; the scale bar denotes $400 \mathrm{~nm}$ and the colours denote different domain orientations from different crystal domains. d, HAADF-STEM image of a cross-section of an $N=10 \mathrm{r}-\mathrm{MoS}_{2}$ film on $\mathrm{AlO}_{x}$ coated with $\mathrm{Al}$, with an interlayer spacing of $6.4 \AA$. e, Large-area $\mathrm{MoS}_{2}$ films transferred onto 1-inch diameter fused silica substrates.
The transmission electron microscopy (TEM) diffraction (Fig.1c, left) and darkfield (inset) images from a representative $\mathrm{MoS}_{2}$ monolayer show that it comprises large $(D \approx 1 \mu \mathrm{m})$, randomly oriented crystalline domains, which connect laterally to form a continuous polycrystalline film. The vacuum stacking generates $r$-TMD films with a precise layer number $(N)$ and high-quality interfaces ${ }^{20}$ with interlayer rotation at every stacked interface. The TEM diffraction pattern of $N=10 \mathrm{r}-\mathrm{MoS}_{2}$ (Fig. 1c, right) shows a ring-like pattern due to the significant increase in the number of diffraction spots, emphasizing the random crystalline orientation in the through-plane direction. Clean and well-defined interfaces can be seen from the cross-sectional high-angle annular darkfield scanning TEM (HAADF-STEM) images of $\mathrm{r}-\mathrm{MoS}_{2}$ (Fig. 1d and Extended Data Fig. 1; see Methods). The monolayers have a uniform interlayer spacing $d \approx 6.4 \AA$ (see Methods and Extended Data Fig. 2), which is close to the expected value ( $6.5 \AA$ ) for twisted $\mathrm{MoS}_{2}$ multilay$\mathrm{ers}^{21}$. Both the growth and stacking steps are scalable, as shown by the optical images of $N=1$ and $N=10 \mathrm{r}-\mathrm{MoS}_{2}$ films $\left(\sim \mathrm{cm}^{2}\right)$ in Fig. $1 \mathrm{e}$ and as demonstrated later in Fig. 4. The large-scale uniformity of these films also enables precise and reproducible measurements with minimal spatial variation (Extended Data Fig. 3a-c). In our experiments, $\mathrm{r}-\mathrm{MoS}_{2}$ or $\mathrm{r}-\mathrm{WS}_{2}$ films with different $N$ (up to 22) are transferred onto a sapphire wafer for the measurements of $\kappa_{\perp}$ or suspended over a holey TEM grid (Fig. 3a) for the measurements of $\kappa_{\|}$.

\section{Ultralow out-of-plane conductivity}

In Fig. 2, we illustrate $\kappa_{\perp}$ of r-TMD films, which is measured using time domain thermoreflectance (TDTR; Fig. 2a, inset; see Methods). A stream of laser pulses (pump) heats up the surface of an Al pad deposited on an $r$-TMD film on sapphire and produces a temperature-sensitive thermoreflectance signal ( $-V_{\text {in }} / V_{\text {out }}$ in Fig. 2a), which is measured with a probe pulse after a varying time delay (for cooling). Figure 2 a shows three representative curves measured from $r-M_{0} S_{2}$ with $N=1,2$ and 10 . The curves flatten with increasing $N$, suggesting that heat dissipation slows down significantly. Fitting these curves using a heat diffusion model (solid lines, Fig. 2a) enables us to obtain $R_{\mathrm{TDTR}}$, the total thermal resistance between the Al transducer layer and sapphire across the r-TMD film for different $N$.
Figure 2b shows $R_{\mathrm{TDTR}}$ versus $N$ for $\mathrm{r}-\mathrm{MoS}_{2}$ ( $N \leq 22$; solid circles) and $\mathrm{r}-\mathrm{WS}_{2}(N \leq 10$; open circles) measured under ambient conditions. We make two observations. First, $R_{\mathrm{TDTR}}$ monotonically increases with $N$. Second, $R_{\mathrm{TDTR}}$ varies linearly with $N$ for $N \geq 2$. These observations confirm that the through-plane thermal transport in r-TMD films is diffusive in nature, in contrast to the ballistic transport reported in few-layer single-crystalline $\mathrm{MoS}_{2}$ (as thick as $\left.240 \mathrm{~nm}\right)^{22,23}$. A single parameter $\kappa_{\perp}$ characterizes the thermal resistance across $\mathrm{r}-\mathrm{MoS}_{2}$ (or $\mathrm{r}-\mathrm{WS}_{2}$ ) using the equation $R_{\mathrm{TDTR}}=R_{0}+N d / \kappa_{\perp}$, where $N d$ is the total film thickness, and $R_{0}$ is a constant corresponding to the total interface resistance ( $\mathrm{r}-\mathrm{TMD} / \mathrm{Al}$ and r-TMD/sapphire; see Extended Data Table 1). Therefore, we apply linear fitting to the data $(N \geq 2)$ in Fig. $2 \mathrm{~b}$ (solid lines) to determine $\kappa_{\perp}$ of $\mathrm{r}-\mathrm{MoS}_{2}$ alone, regardless of the quality and chemical nature of the top and bottom interfaces (see Methods and Extended Data Fig. 3d), which can potentially be altered by metal deposition ${ }^{24,25}$. We measure $\kappa_{\perp}=57 \pm 3 \mathrm{~mW} \mathrm{~m}^{-1} \mathrm{~K}^{-1}$ for $\mathrm{r}-\mathrm{MoS}_{2}$ and $\kappa_{\perp}=41 \pm 3 \mathrm{~mW} \mathrm{~m}^{-1} \mathrm{~K}^{-1}$ for $\mathrm{r}-\mathrm{WS}_{2}$, which are similar to the lowest value ever observed in a fully dense solid ${ }^{15}$ and comparable to the thermal conductivity of ambient air $\left(-26 \mathrm{~mW} \mathrm{~m}^{-1} \mathrm{~K}^{-1}\right)$. These values are approximately two orders of magnitude smaller than those of single-crystalline $\mathrm{MoS}_{2}\left(2-5 \mathrm{~W} \mathrm{~m}^{-1} \mathrm{~K}^{-1}\right)^{26,27}$ or $\mathrm{WS}_{2}\left(\sim 3 \mathrm{~W} \mathrm{~m}^{-1} \mathrm{~K}^{-1}\right)^{27}$, despite the r-TMD films having the same chemical composition as their bulk counterparts as well as clean interfaces (Fig. 1d). This strongly suggests that the main difference, the interlayer rotation, is the principal cause for the ultralow $\kappa_{\perp}$ in these $\mathrm{r}$-TMD films. Furthermore, repeating similar TDTR experiments on $\mathrm{r}-\mathrm{MoS}_{2}$ at different temperatures $(T)$ produces a relatively flat $\kappa_{\perp}(T)$ curve (green stars, Fig. 2c), a behaviour different from the decreasing $\kappa_{\perp}$ with $T$ seen in bulk $\mathrm{MoS}_{2}$ (blue squares, lower Fig. 2c).

To understand the microscopic mechanisms that give rise to the dramatic reduction in $\kappa_{\perp}$, we carry out homogeneous non-equilibrium molecular dynamics (HNEMD) simulations for the model structures of $\mathrm{r}-\mathrm{MoS}_{2}$ and bulk $\mathrm{MoS}_{2}$ (see Methods and Extended Data Table 2) ${ }^{28-30}$. Figure 2c shows $\kappa_{\|}$and $\kappa_{\perp}$ of $\mathrm{r}-\mathrm{MoS}_{2}$ (solid circles) and bulk $\mathrm{MoS}_{2}$ (empty circles) calculated from our molecular dynamics (MD) simulations at different temperatures. The calculated $\kappa_{\perp}$ drops by a factor of more than 20 , from $3.7 \pm 0.5 \mathrm{~W} \mathrm{~m}^{-1} \mathrm{~K}^{-1}$ in bulk $\mathrm{MoS}_{2}$ to $0.16 \pm 0.04 \mathrm{~W} \mathrm{~m}^{-1} \mathrm{~K}^{-1}$ in r- $\mathrm{MoS}_{2}$ at $300 \mathrm{~K}$, and also does not decrease with $T$, suggesting a transition away from the phonon-limited thermal transport mechanism observed in bulk $\operatorname{MoS}_{2}$. 

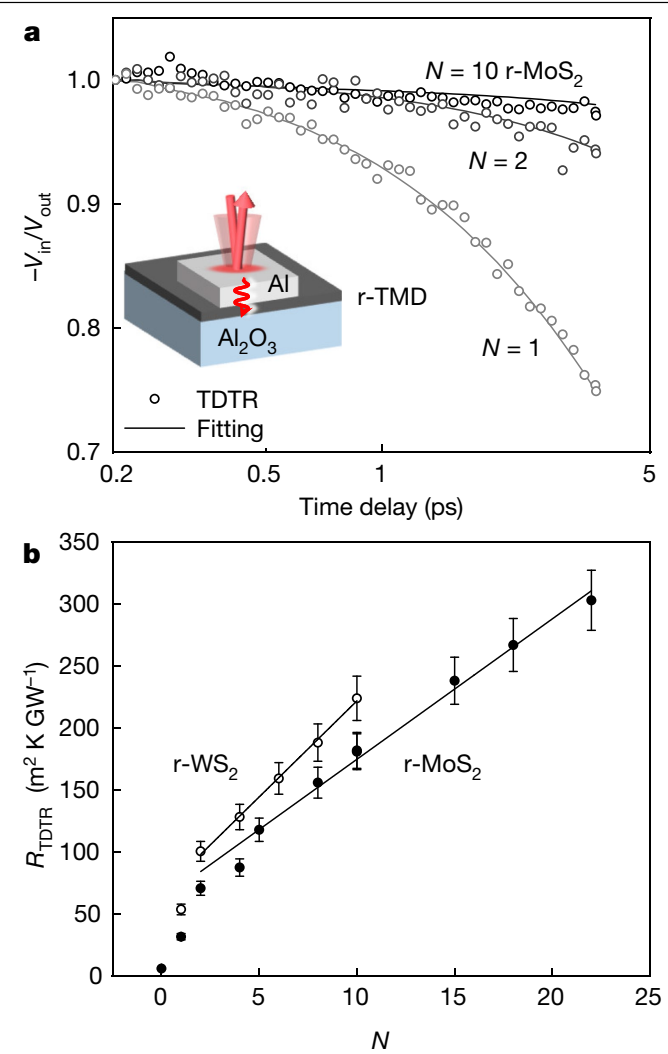

Fig. 2 |Through-plane thermal properties of $\mathbf{r}-\mathrm{MoS}_{\mathbf{2}} \cdot \mathbf{a}$, TDTR heat dissipation curves of $N$-layer $\mathrm{r}-\mathrm{MoS}_{2}$ films. Inset: TDTR sample geometry. b, Measured thermal resistances across r-TMD films, where the error bars are the TDTR measurement uncertainties. The thermal conductivities for $\mathrm{r}-\mathrm{MoS}_{2}$ and $\mathrm{r}-\mathrm{WS}_{2}$ are calculated from the slope using the formula $R_{\mathrm{TDTR}}=R_{0}+\mathrm{Nd} / \kappa_{\perp}$, whereby $R_{0}$ is the total interfacial thermal resistance.c, Experiment and MD simulation results of $\kappa(T)$ of $\mathrm{MoS}_{2}$ and r-MoS films. The error bars to the MD
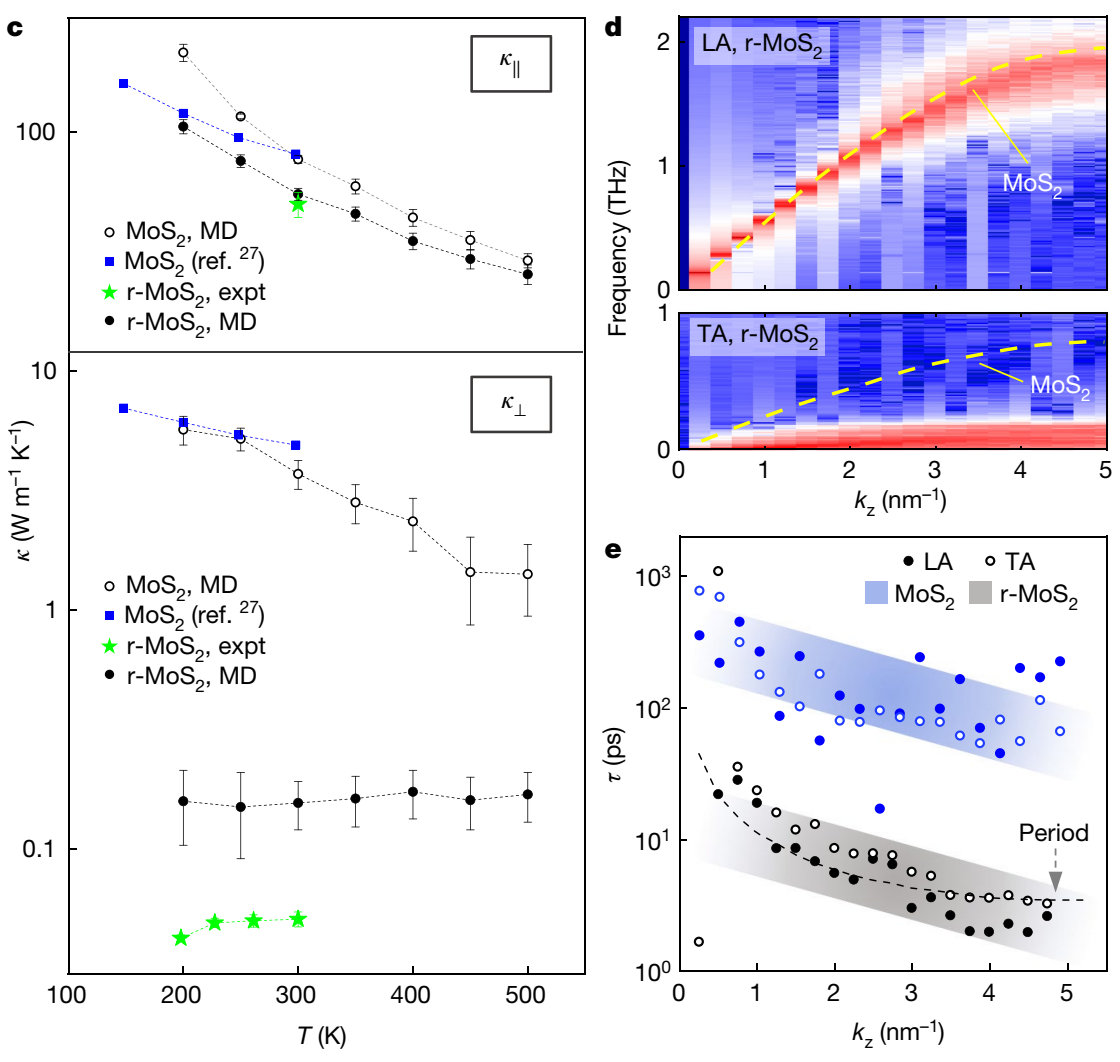

simulations originate from the simulation uncertainties. The dotted lines connecting the individual data points are guides to the eye. d, LA (top) and $\mathrm{TA}$ (bottom) phonon dispersion curves of $\mathrm{r}-\mathrm{MoS}_{2}$ along the $\Gamma-\mathrm{A}$ direction. The dotted lines denote the acoustic curves corresponding to bulk $\mathrm{MoS}_{2}$. e, Lifetime of $\mathrm{LA}$ and TA phonons parallel to the $\mathrm{\Gamma -A}$ direction in bulk and $\mathrm{r}-\mathrm{MoS}_{2}$. The dashed line is the LA mode vibration period derived from the dispersion curve in d.

Further analysis of the vibrational spectrum of $\mathrm{r}-\mathrm{MoS}_{2}$ enables us to break down the reduction in $\kappa_{\perp}$ in terms of the changes in the group velocities $\left(v_{\mathrm{g}}\right)$ and lifetimes $(\tau)$, which are the two factors that determine the thermal conductivity according to Boltzmann transport theory. Figure $2 \mathrm{~d}$ shows that the $v_{\mathrm{g}}$ of the through-plane longitudinal acoustic (LA) mode in $\mathrm{r}-\mathrm{MoS}_{2}$ remains similar to that of bulk $\mathrm{MoS}_{2}$ (dashed lines), but the transverse acoustic (TA) modes in $\mathrm{r}-\mathrm{MoS}_{2}$ undergo extreme softening with their $v_{\mathrm{g}}$ s practically vanishing ${ }^{31}$. This implies a loss of resistance with respect to lateral shear, consistent with the low-frequency Raman spectra of $\mathrm{r}-\mathrm{MoS}_{2}$ films (see Methods and Extended Data Fig. 4) and previous calculations ${ }^{32,33}$. In addition, the $\tau$ of both the LA and the TA modes (Fig. 2e) in r-MoS are more than one order of magnitude smaller than in bulk $\mathrm{MoS}_{2}$, with the LA lifetimes being close to the period of the LA mode vibration (dashed line). From these results, the median mean free path $\tilde{l}=v_{\mathrm{g}} \tau$ for the LA modes is estimated to be $2 \mathrm{~nm}$, suggesting that the heat-carrying LA modes are strongly scattered and that a larger $D$ is unlikely to significantly affect $\kappa_{\perp}$ since $D>>\tilde{l}$. Overall, the strongly suppressed TA modes, indicating a loss of resistance to lateral shear, and the overdamping of the LA modes as the main heat carriers, lead to extremely inefficient thermal transport along the through-plane direction in $\mathrm{r}-\mathrm{MoS}_{2}$. Along with the nearly temperature-independent $\boldsymbol{\kappa}_{\perp}$, this result suggests a glass-like conduction mechanism.

\section{In-plane conductivity and anisotropy}

In contrast to $\kappa_{\perp}, \kappa_{\|}$remains high in our simulations with only a modest reduction compared to the ideal bulk crystal (less than a factor of two at $300 \mathrm{~K}$; Fig. 2c). This is indeed what we observe in our Raman thermometry experiments as discussed in Fig. 3 (see Methods). We direct a focused laser spot $(\lambda=532 \mathrm{~nm})$ at the centre of a suspended $\mathrm{r}-\mathrm{MoS}_{2}$ film (Fig. 3a; hole diameter of $5 \mu \mathrm{m}$, at 15 torr), which increases its temperature $(\Delta T)$ locally upon absorbing laser power $P_{\text {abs. }} \Delta T$ is then measured using the temperature-sensitive Raman peak shift $(\Delta \omega)$ using a sensitivity factor $(|\mathrm{d} \omega / \mathrm{d} T|)$ independently measured for each sample. Examples of Raman spectra measured for $N=2$ are shown in Fig. $3 \mathrm{~b}$.

Figure 3c plots $\Delta \omega$ versus $P_{\text {abs }}$ for r-MoS $\mathrm{MoS}_{2}$ with different $N(2$ to 5$)$. The slope of the linear fit $\left(\left|\mathrm{d}(\Delta \omega) / \mathrm{d} P_{\text {abs }}\right|\right)$, which is inversely proportional to the in-plane thermal conductance of the film, is plotted in the inset (solid dots; $D \approx 1 \mu \mathrm{m}$ ). We again observe a linear relation, which indicates that $\kappa_{\|}$is well defined for $\mathrm{r}-\mathrm{MoS}_{2}$ independent of $N$, similar to the case of $\kappa_{\perp}$. Using a simple diffusion model with radial symmetry (see Methods and Extended Data Fig. $5 \mathrm{c}$ for calculation details and other input measurements), we calculate a high $\kappa_{\|}$value of $50 \pm 6 \mathrm{~W} \mathrm{~m}^{-1} \mathrm{~K}^{-1}$. This value is similar to the predictions of our MD simulations (Fig. 2c) and consistent with previous reports of Raman thermometry on single-crystalline monolayer $\mathrm{MoS}_{2}\left(35-84 \mathrm{~W} \mathrm{~m}^{-1} \mathrm{~K}^{-1}\right)$ at room temperature ${ }^{34-37}$. The $\boldsymbol{K}_{\|}$of these r- $\mathrm{MoS}_{2}$ films is close to the intrinsic phonon-limited value despite the films being made of polycrystalline monolayers. This result is further supported by our additional measurements on continuous $r-\mathrm{MoS}_{2}$ films with a smaller $D \approx 400 \mathrm{~nm}$ (open dots, dashed lines, Fig. 3c inset; Extended Data Fig. 5e). The measured value of $\kappa_{\|} \approx 44 \pm 6 \mathrm{~W} \mathrm{~m}^{-1} \mathrm{~K}^{-1}$ is within the margin of error of that of the $D \approx 1 \mu \mathrm{m}$ films. This suggests that the phonon mean free path is smaller than $400 \mathrm{~nm}$, which is consistent with previous reports $\mathrm{s}^{23,38-42}$. Furthermore, the measured in-plane conductance decreases with $T$ (Extended Data Fig. 6a). This further confirms the phonon-mediated 

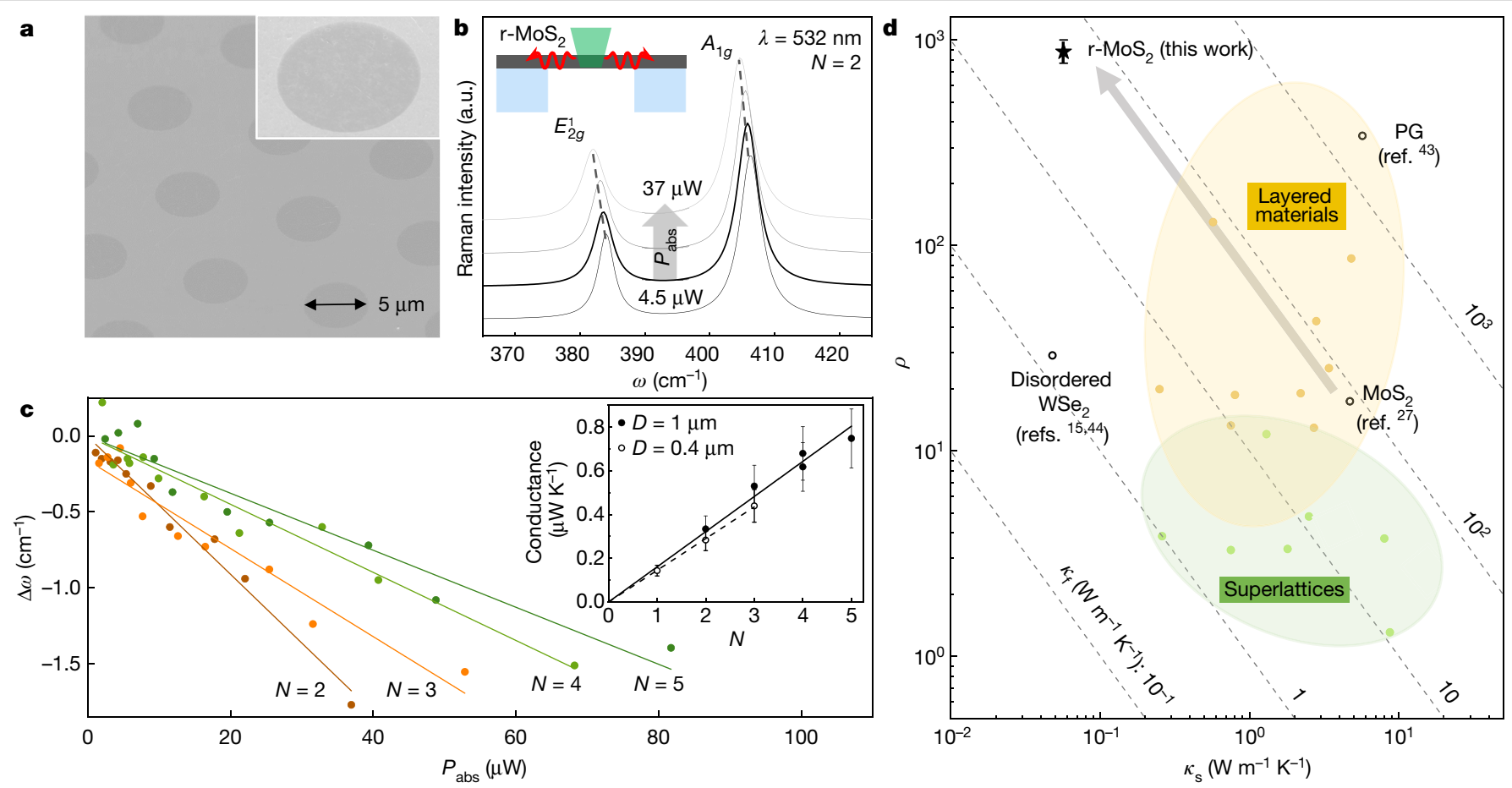

Fig. 3 | In-plane thermal properties and thermal anisotropy of $\mathbf{r}-\mathrm{MoS}_{\mathbf{2}}$ films. a, $45^{\circ} \mathrm{SEM}$ micrograph of an $N=4 \mathrm{r}-\mathrm{MoS}_{2}$ film suspended on a TEM grid for Raman thermometry. b, Raman spectra of an $N=2 \mathrm{r}-\mathrm{MoS}_{2}$ film with different absorbed laser powers. Inset: Raman thermometry sample geometry.c, $\mathrm{A}_{1 g}$ Raman peak shifts versus power absorbed by $\mathrm{r}-\mathrm{MoS}_{2}$ films of various $N$. Inset: layer-dependent thermal conductance values (absorbed power divided by temperature increase) in domain size $D=1 \mu \mathrm{m}$ and

thermal transport mechanism in-plane, in contrast to the glass-like thermal conduction along the through-plane direction.

Our experiments and calculations confirm that interlayer rotation in r-TMD films results in highly directional thermal conductivity and a direction-dependent thermal conduction mechanism. The rotation significantly reduces $\kappa_{\perp}$ while maintaining high $\kappa_{\|}$, leading to an ultrahigh value of $\rho$. We estimate $\rho \approx 880 \pm 110$ at room temperature for ther-MoS films, higher than that of pyrolytic graphite (PG), which is considered to be one of the most anisotropic thermal conductors $(\rho \approx 340)^{43}$. In Fig. 3d, we compare our result with other previously reported values of $\rho$ in phononbased solids ${ }^{15,27,43,44}$ (for a full comparison, see Extended Data Fig. 6b). Compared to a bulk $\mathrm{MoS}_{2}$ crystal $(\rho \approx 20)^{27}$ or disordered layered $\mathrm{WSe}_{2}$ $(\rho \approx 30)^{15,44}, \mathrm{r}-\mathrm{MoS}_{2}$ has a significantly larger $\rho$ because interlayer rotation reduces only $\kappa_{\perp}$, as denoted by the grey arrow parallel to the equi- $\kappa_{\mathrm{f}}$ lines. This also suggests that $\rho$ can be made even larger by starting with the monolayers of a layered vdW material with a higher $\kappa_{\|}$value such as graphene.

\section{Anisotropic vdW heat diffuser}

In Fig. 4, we show that the extreme anisotropy of our $\mathrm{r}-\mathrm{MoS}_{2}$ films can lead to excellent heat dissipation in-plane from a heat source and drastic thermal insulation in the through-plane direction. Using the COMSOL software, we perform thermal finite-element simulations of a $10-\mathrm{nm}$ thick r-MoS film draped over a nanoscale Au electrode (15 $\mathrm{nm}$ tall, $100 \mathrm{~nm}$ wide) on a $50 \mathrm{~nm} \mathrm{SiO} / 2$ Si substrate (Fig. 4a). Our simulation results show that for a fixed power of $8 \mathrm{~mW}$ supplied to the Au electrode (near thermal breakdown), the temperature rise $\Delta T$ of the Au electrode covered by $\mathrm{r}-\mathrm{MoS}_{2}$ is $50 \mathrm{~K}$ lower than that of the bare electrode, thereby demonstrating our film's effectiveness at spreading heat due to its excellent $\boldsymbol{K}_{\| \mid}$(Fig. 4b, c). Interestingly, the extreme thermal anisotropy of our $\mathrm{r}-\mathrm{MoS}_{2}$ films provides thermal insulation in the through-plane direction,
$D=400 \mathrm{~nm} \mathrm{r}-\mathrm{MoS}_{2}$ films. The error bars are the propagated uncertainties from the calculation of the conductance value for each $N$.d, Comparison of $\rho$ ( $y$ axis), $\kappa_{\mathrm{s}}\left(x\right.$ axis), and $\kappa_{\mathrm{f}}$ (diagonal dashed lines) measured for different anisotropic thermal conductors. $r-\mathrm{MoS}_{2}$ has an ultrahigh $\rho$ close to 900 , which is larger than bulk $\mathrm{MoS}_{2}, \mathrm{PG}$, and disordered layered WSe $\mathrm{W}_{2}$. The error bar for $\rho$ of r-MoS $\mathrm{Momes}_{2}$ from the propagated uncertainties of the calculated $\kappa_{\perp}$ and $\kappa_{\|}$ values.

with much lower $\mathrm{MoS}_{2}$ surface $\Delta T$ values that are only one-third of the value of the bare Au electrode. While single-crystal $\mathrm{MoS}_{2}$ displays similar properties, the insulation effect is stronger in $\mathrm{r}-\mathrm{MoS}_{2}$ (Extended Data Fig. 7a). This implies that heat is efficiently directed away from the hot Au electrode laterally through $\mathrm{r}-\mathrm{MoS}_{2}$ but not to the surface of $\mathrm{r}-\mathrm{MoS}_{2}$, making the surface of the entire device significantly cooler.

Our experiments corroborate these simulation results. For this, we fabricate nanoscale Au electrodes with the same geometry and substrate as in our simulation (image shown in Fig. 4d, inset) and transfer $N=16$ $\mathrm{r}-\mathrm{MoS}_{2}(\sim 10 \mathrm{~nm}$ thick$)$ using the vacuum stacking process. Both bare and coated Au electrodes show similar resistance at low currents. At higher currents, current-induced Joule heating leads to the thermally activated electromigration process, which causes the electrodes to fail ${ }^{45}$. Figure $4 \mathrm{~d}$ compares representative current-voltage $(I-V)$ curves measured from a bare and coated Au electrode, which shows that the Au electrode with $\mathrm{r}-\mathrm{MoS}_{2}$ can carry a larger current without breaking. The histogram of critical current $I_{\mathrm{c}}$ (maximum current a Au electrode sustains for at least $20 \mathrm{~s}$ ) measured from 20 electrodes ( 10 bare and 10 with $r-M_{2} S_{2}$ ) reveals a $~ 50 \%$ increase in the median $I_{\mathrm{c}}$ values (Fig. $4 \mathrm{e}$ ). These results demonstrate

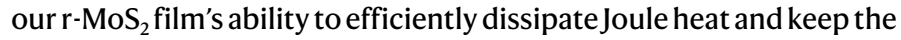
electrodes cool, as our simulation predicts. As the electromigration process is dominated by the temperature, the observed increase of $I_{\mathrm{c}}$ and maximum power before breaking is in good agreement with our

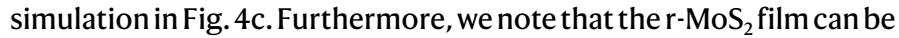
integrated with the Au electrodes using mild conditions that do not affect their electrical properties (Extended Data Fig. 7b).

\section{Outlook}

We expect interlayer rotation to be an effective and generalizable way to reduce $\kappa_{\perp}$ and potentially engineer anisotropic thermal properties 


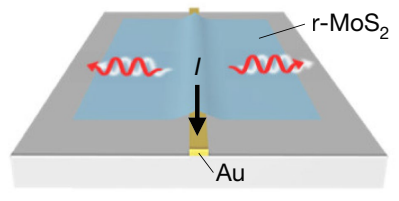

b
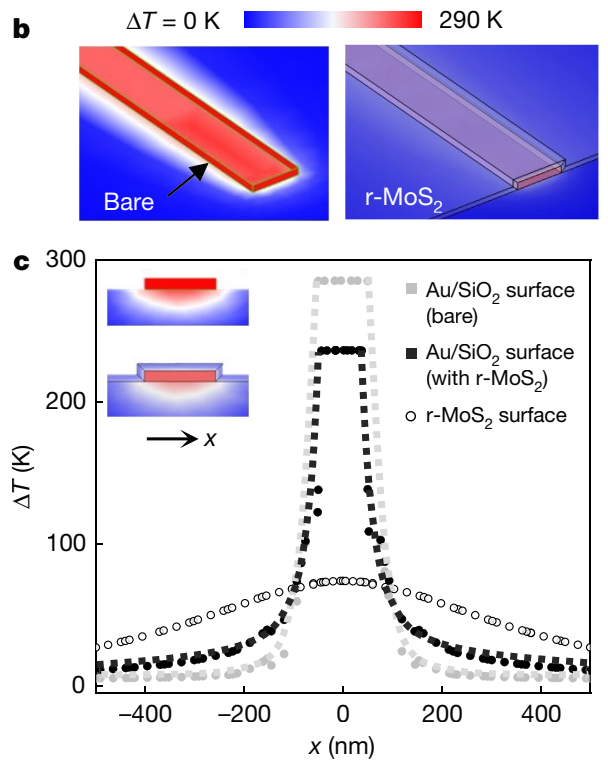

Fig. 4 | Temperature profiles and heat spreader efficiencies of $\mathbf{r}-\mathrm{MoS}_{\mathbf{2}}$ films on Au electrodes. a, Schematic of the sample configuration of r-MoS${ }_{2}$ draped across a current-carrying Au electrode that is $100 \mathrm{~nm}$ wide, $15 \mathrm{~nm}$ thick and $10 \mu \mathrm{m}$ long. b. Thermal finite element modelling results of Au electrodes (bare, covered with 10 -nm-thick $\mathrm{r}-\mathrm{MoS}_{2}$ ) at constant heating power of $8 \mathrm{~mW}$ supplied through Joule heating. $\mathbf{c}$, Lateral profiles of temperature increases across the

in a variety of layered materials. Our results call for a systematic study of the exact relation between $\kappa_{\perp}$ and rotation angle, which could reveal unexpected relationships analogous to the studies of electrical transport in twisted bilayer graphene ${ }^{46}$. Interlayer rotations can be combined with other parameters (such as pressure or interlayer spacing ${ }^{47,48}$ ) and advanced structures (superlattices and heterostructures ${ }^{18}$ ) to realize highly tunable $\rho$, allowing for the customization of thermal transport properties with an unprecedented level of directional and spatial control.

\section{Online content}

Any methods, additional references, Nature Research reporting summaries, source data, extended data, supplementary information, acknowledgements, peer review information; details of author contributions and competing interests; and statements of data and code availability are available at https://doi.org/10.1038/s41586-021-03867-8.

1. Minnich, A. J. Exploring the extremes of heat conduction in anisotropic materials. Nanoscale Microscale Thermophys. Eng. 20, 1-21 (2016).

2. Cui, Y., Li, M. \& Hu, Y. Emerging interface materials for electronics thermal management: experiments, modeling, and new opportunities. J. Mater. Chem. C 8, 10568-10586 (2020).

3. Moore, A. L. \& Shi, L. Emerging challenges and materials for thermal management of electronics. Mater. Today 17, 163-174 (2014).

4. Norley, J., Tzeng, J. J.-W., Getz, G., Klug, J. \& Fedor, B. The development of a natural graphite heat-spreader. In Seventeenth Annual IEEE Semiconductor Thermal Measurement and Management Symposium 107-110 (IEEE, 2001).

5. Chung, D. D. L. \& Takizawa, Y. Performance of isotropic and anisotropic heat spreaders J. Electron. Mater. 41, 2580-2587 (2012).

6. Termentzidis, K. Thermal conductivity anisotropy in nanostructures and nanostructured materials. J. Phys. Appl. Phys. 51, 094003 (2018).
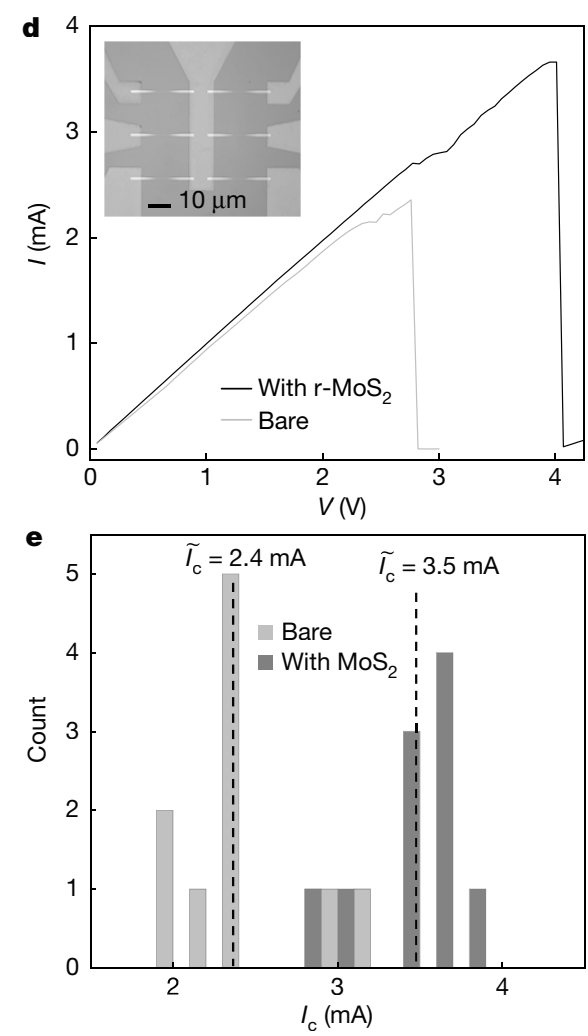

$\mathrm{Au} / \mathrm{SiO}_{2}$ surface (solid dots) and on the $\mathrm{r}-\mathrm{MoS}_{2}$ top surface (open circles). Insets: cross-sectional temperature distribution of Au electrodes with and without $\mathrm{r}-\mathrm{MoS}_{2}$, using the same colour scale as in b. d, $I-V$ curve of an Au electrode, with and without $N=16 \mathrm{r}-\mathrm{MoS}_{2}$. Inset: optical micrograph of six fabricated $\mathrm{Au}$ electrodes. e, Histogram of $I_{\mathrm{c}}$ of Au electrodes with and without an $N=16 \mathrm{r}-\mathrm{MoS}_{2}$ heat spreader and their median values. superlattices. Appl. Phys. Lett. 70, 2957-2959 (1997).

8. Luckyanova, M. N. et al. Anisotropy of the thermal conductivity in GaAs/AlAs superlattices. Nano Lett. 13, 3973-3977 (2013).

9. Borca-Tasciuc, T. et al. Thermal conductivity of symmetrically strained $\mathrm{Si} / \mathrm{Ge}$ superlattices. Superlattices Microstruct. 28, 199-206 (2000).

10. Lee, W.-Y. et al. Anisotropic temperature-dependent thermal conductivity by an $\mathrm{Al}_{2} \mathrm{O}_{3}$ interlayer in $\mathrm{Al}_{2} \mathrm{O}_{3} / \mathrm{ZnO}$ superlattice films. Nanotechnology 28, 105401 (2017).

11. Rawat, V., Koh, Y. K., Cahill, D. G. \& Sands, T. D. Thermal conductivity of (Zr,W)N/ScN metal/semiconductor multilayers and superlattices. J. Appl. Phys. 105, 024909 (2009).

12. Sun, B. et al. Dislocation-induced thermal transport anisotropy in single-crystal group-III nitride films. Nat. Mater. 18, 136-140 (2019).

13. Jiang, P., Qian, X., Yang, R. \& Lindsay, L. Anisotropic thermal transport in bulk hexagonal boron nitride. Phys. Rev. Mater. 2, 64005 (2018).

14. Chiritescu, C. et al. Low thermal conductivity in nanoscale layered materials synthesized by the method of modulated elemental reactants. J. Appl. Phys. 104 033533 (2008).

15. Chiritescu, C. et al. Ultralow thermal conductivity in disordered, layered WSe $\mathrm{C}_{2}$ crystals. Science 315, 351-353 (2007).

16. Hadland, E. C. et al. Ultralow thermal conductivity of turbostratically disordered $\mathrm{MoSe}_{2}$ ultra-thin films and implications for heterostructures. Nanotechnology 30, 285401 (2019).

17. Hadland, E. et al. Synthesis, characterization, and ultralow thermal conductivity of a lattice-mismatched $\mathrm{SnSe}_{2}\left(\mathrm{MoSe}_{2}\right)_{1.32}$ heterostructure. Chem. Mater. 31, 5699-5705 (2019).

18. Vaziri, S. et al. Ultrahigh thermal isolation across heterogeneously layered two-dimensional materials. Sci. Adv. 5, eaax1325 (2019).

19. Kang, K. et al. High-mobility three-atom-thick semiconducting films with wafer-scale homogeneity. Nature 520, 656-660 (2015).

20. Kang, K. et al. Layer-by-layer assembly of two-dimensional materials into wafer-scale heterostructures. Nature 550, 229-233 (2017).

21. Liu, K. et al. Evolution of interlayer coupling in twisted molybdenum disulfide bilayers. Nat. Commun. 5, 4966 (2014)

22. Sood, A. et al. Quasi-ballistic thermal transport across $\mathrm{MoS}_{2}$ thin films. Nano Lett. 19 2434-2442 (2019).

23. $\mathrm{Li}, \mathrm{Z}$. et al. Size dependence and ballistic limits of thermal transport in anisotropic layered two-dimensional materials. Preprint at arXiv https://arxiv.org/abs/1711.02772 (2017). 
24. Schauble, K. et al. Uncovering the effects of metal contacts on monolayer $\mathrm{MoS}_{2}$. ACS Nano 14, 14798-14808 (2020).

25. Liu, Y. et al. Approaching the Schottky-Mott limit in van der Waals metal-semiconductor junctions. Nature 557, 696-700 (2018).

26. Liu, J., Choi, G.-M. \& Cahill, D. G. Measurement of the anisotropic thermal conductivity of molybdenum disulfide by the time-resolved magneto-optic Kerr effect. J. Appl. Phys. 116, 233107 (2014).

27. Jiang, P., Qian, X., Gu, X. \& Yang, R. Probing anisotropic thermal conductivity of transition metal dichalcogenides $\mathrm{MX}_{2}(\mathrm{M}=\mathrm{Mo}, \mathrm{W}$ and $\mathrm{X}=\mathrm{S}, \mathrm{Se})$ using time-domain thermoreflectance. Adv. Mater. 29, 1701068 (2017).

28. Evans, D. J. Homogeneous NEMD algorithm for thermal conductivity-application of non-canonical linear response theory. Phys. Lett. A 91, 457-460 (1982).

29. Fan, Z., Chen, W., Vierimaa, V. \& Harju, A. Efficient molecular dynamics simulations with many-body potentials on graphics processing units. Comput. Phys. Commun. 218, 10-16 (2017).

30. $\mathrm{Xu}, \mathrm{K}$. et al. Thermal transport in $\mathrm{MoS}_{2}$ from molecular dynamics using different empirical potentials. Phys. Rev. B 99, 054303 (2019).

31. Li, D., Schleife, A., Cahill, D. G., Mitchson, G. \& Johnson, D. C. Ultralow shear modulus of incommensurate $[\mathrm{SnSe}]_{n}\left[\mathrm{MoSe}_{2}\right]_{n}$ layers synthesized by the method of modulated elemental reactants. Phys. Rev. Mater. 3, 043607 (2019).

32. Huang, S. et al. Probing the interlayer coupling of twisted bilayer $\mathrm{MoS}_{2}$ using photoluminescence spectroscopy. Nano Lett. 14, 5500-5508 (2014).

33. Erhart, P., Hyldgaard, P. \& Lindroth, D. O. Microscopic origin of thermal conductivity reduction in disordered van der Waals solids. Chem. Mater. 27, 5511-5518 (2015).

34. Yan, R. et al. Thermal conductivity of monolayer molybdenum disulfide obtained from temperature-dependent Raman spectroscopy. ACS Nano 8, 986-993 (2014).

35. Sahoo, S., Gaur, A. P. S., Ahmadi, M., Guinel, M. J.-F. \& Katiyar, R. S. Temperature-dependent Raman studies and thermal conductivity of few-layer $\mathrm{MoS}_{2}$. J. Phys. Chem. C 117, 9042-9047 (2013).

36. Zhang, X. et al. Measurement of lateral and interfacial thermal conductivity of single- and bilayer $\mathrm{MoS}_{2}$ and $\mathrm{MoSe}_{2}$ using refined optothermal Raman technique. ACS Appl. Mater. Interfaces 7, 25923-25929 (2015).

37. Taube, A., Judek, J., Łapińska, A. \& Zdrojek, M. Temperature-dependent thermal properties of supported $\mathrm{MoS}_{2}$ monolayers. ACS Appl. Mater. Interfaces 7, 5061-5065 (2015).

38. Lindroth, D. O. \& Erhart, P. Thermal transport in van der Waals solids from first-principles calculations. Phys. Rev. B 94, 115205 (2016).
39. Jo, I., Pettes, M. T., Ou, E., Wu, W. \& Shi, L. Basal-plane thermal conductivity of few-layer molybdenum disulfide. Appl. Phys. Lett. 104, 201902 (2014).

40. Wei, X. et al. Phonon thermal conductivity of monolayer $\mathrm{MoS}_{2}$ : a comparison with single layer graphene. Appl. Phys. Lett. 105, 103902 (2014).

41. Cai, Y., Lan, J., Zhang, G. \& Zhang, Y.-W. Lattice vibrational modes and phonon thermal conductivity of monolayer $\mathrm{MoS}_{2}$. Phys. Rev. B 89, 035438 (2014).

42. Liu, X., Zhang, G., Pei, Q.-X. \& Zhang, Y.-W. Phonon thermal conductivity of monolayer $\mathrm{MoS}_{2}$ sheet and nanoribbons. Appl. Phys. Lett. 103, 133113 (2013).

43. Ho, C. Y., Powell, R. W. \& Liley, P. E. Thermal conductivity of the elements. J. Phys. Chem. Ref. Data 1, 279-421 (1972).

44. Mavrokefalos, A., Nguyen, N. T., Pettes, M. T., Johnson, D. C. \& Shi, L. In-plane thermal conductivity of disordered layered $\mathrm{WSe}_{2}$ and $(\mathrm{W})_{x}\left(\mathrm{WSe}_{2}\right)_{y}$ superlattice films. Appl. Phys. Lett. 91, 171912 (2007).

45. Ho, P. \& Kwok, T. Electromigration in metals. Rep. Prog. Phys. 52, 301-348 (1989).

46. Cao, Y. et al. Unconventional superconductivity in magic-angle graphene superlattices. Nature 556, 43-50 (2018).

47. Sood, A. et al. An electrochemical thermal transistor. Nat. Commun. 9, 4510 (2018).

48. Chen, S., Sood, A., Pop, E., Goodson, K. E. \& Donadio, D. Strongly tunable anisotropic thermal transport in $\mathrm{MoS}_{2}$ by strain and lithium intercalation: first-principles calculations. 2D Mater. 6, 025033 (2019).

Publisher's note Springer Nature remains neutral with regard to jurisdictional claims in published maps and institutional affiliations.

Open Access This article is licensed under a Creative Commons Attribution 4.0 International License, which permits use, sharing, adaptation, distribution and reproduction in any medium or format, as long as you give appropriate credit to the original author(s) and the source, provide a link to the Creative Commons license, and indicate if changes were made. The images or other third party material in this article are included in the article's Creative Commons license, unless indicated otherwise in a credit line to the material. If material is not included in the article's Creative Commons license and your intended use is not permitted by statutory regulation or exceeds the permitted use, you will need to obtain permission directly from the copyright holder. To view a copy of this license, visit http://creativecommons.org/licenses/by/4.0/.

(c) The Author(s) 2021 


\section{Methods}

\section{Sample preparation}

Large-area, polycrystalline transition metal dichalcogenide (TMD) $\left(\mathrm{MoS}_{2}\right.$ and $\left.\mathrm{WS}_{2}\right)$ monolayers were grown on $\mathrm{SiO}_{2} / \mathrm{Si}$ substrates in a hot-walled tube furnace via metal-organic chemical vapour deposition adapted from a previously reported protocol ${ }^{19}$. The growth conditions were optimized to produce high-quality monolayer materials with structural characteristics necessary for thermal measurements. These characteristics include large domain size $(D \approx 1 \mu \mathrm{m}$ and $0.4 \mu \mathrm{m})$, full monolayer coverage, and laterally stitched grain boundaries.

Briefly, $\mathrm{Mo}(\mathrm{CO})_{6}$ and $\mathrm{W}(\mathrm{CO})_{6}$ (diluted in $\mathrm{N}_{2}$ to 15 torr) were used as the metal precursors for the $\mathrm{MoS}_{2}$ and WS 2 growths, respectively. $\left(\mathrm{C}_{2} \mathrm{H}_{5}\right)_{2} \mathrm{~S}$ was used as the chalcogen source. All precursors were kept at room temperature. $\mathrm{N}_{2}$ and $\mathrm{H}_{2}$ were used as carrier gases. Typical growth times were $15-20 \mathrm{~h}$ for $\mathrm{MoS}_{2}$ at a growth temperature of $525^{\circ} \mathrm{C}$. Typical growth times for $\mathrm{WS}_{2}$ were $2 \mathrm{~h}$ at a temperature of $650^{\circ} \mathrm{C}$.

To make the r-TMD films, a TMD monolayer was spin-coated with PMMA A8 (poly-methyl methacrylate, $495 \mathrm{~K}, 4 \%$ diluted in anisole) at $2,800 \mathrm{rpm}$ for $60 \mathrm{~s}$, then baked at $180^{\circ} \mathrm{C}$ for $3 \mathrm{~min}$. The PMMA-coated monolayer was stacked onto TMD monolayers layer by layer to a target layer number $(N)$ and transferred to the desired substrates using a previously reported programmed vacuum stacking method ${ }^{20}$.

TDTR samples. The stacked r-TMD films were transferred onto sapphire substrates (Valley Design, C-plane), which were cleaned with Nanostrip solution for $20 \mathrm{~min}$ at $60^{\circ} \mathrm{C}$ and then rinsed with deionized water. The PMMA layer on the film was removed by immersing the entire substrate in acetone at $60^{\circ} \mathrm{C}$ for $1 \mathrm{~h}$. The film was annealed under a $400 / 100 \mathrm{SCCM}$ $\mathrm{Ar} / \mathrm{H}_{2}$ environment at $350^{\circ} \mathrm{C}$ for $4 \mathrm{~h}$. After cleaning, $-90-\mathrm{nm}$-thick, $90 \mu \mathrm{m} \times 90 \mu \mathrm{m} \mathrm{Al}$ pads were deposited onto the TMD films through a holey TEM grid shadow mask using electron-beam evaporation.

Raman thermometry samples. Raman experiments were performed on a different set of films from the TDTR-measured films. First, holey $\mathrm{SiN}_{x}$ transmission electron microscopy (TEM) grids were cleaned in a $\mathrm{N}_{2} / \mathrm{H}_{2}$ plasma at $100^{\circ} \mathrm{C}$ and 180 mtorr for 3 min, followed by the transfer of stacked $\mathrm{MoS}_{2}$ films onto the TEM grids. During the transfer process, the PMMA-coated $\mathrm{r}-\mathrm{MoS}_{2}$ was suspended on holey thermal release tape before contacting the TEM grid. The extra PMMA-MoS $\mathrm{S}_{2}$ not on the TEM grid was cut away at $180^{\circ} \mathrm{C}$ so the PMMA layer was softened. PMMA was removed from $\mathrm{r}-\mathrm{MoS}_{2}$ on the TEM grid via annealing the film in $400 / 100 \mathrm{SCCM} \mathrm{Ar} / \mathrm{H}_{2}$ at $350^{\circ} \mathrm{C}$ for $4 \mathrm{~h}$.

\section{Cross-sectional STEM}

The $N=10$ films were coated with Al that was electron beam evaporated onto the surface, whereas the top surface of $N=20$ films was bare. The $\mathrm{r}-\mathrm{MoS}_{2}$ cross-section was prepared using a Thermo Scientific Strata 400 focused ion beam. Protective layers of carbon $(-200 \mathrm{~nm})$ and platinum $(\sim 1 \mu \mathrm{m})$ were deposited on the sample. A cross-section was milled at a $90^{\circ}$ angle from the sample using a Ga ion beam at $30 \mathrm{kV}$. The cross-section was then polished to $\sim 150 \mathrm{~nm}$ thickness with the ion beam at $5 \mathrm{kV}$.

The cross-section was imaged in a Thermo Scientific Titan Themis scanning transmission electron microscope at $120 \mathrm{kV}$ with a probe convergence angle of $21.4 \mathrm{mrad}$. The $N=10$ film was imaged at a beam voltage of $120 \mathrm{kV}$, whereas the $N=20$ film was imaged at $300 \mathrm{kV}$. All images were analysed using the open-source software Cornell Spectrum Imager ${ }^{49}$. The high-angle annular dark field (HAADF) image of the sample (see 'TMD films with interlayer rotation' in the main text; Fig. 1d) shows, from top to bottom, the Al crystal lattice along the [110] zone axis, ten layers of $\mathrm{MoS}_{2}$ (bright bands), followed by an $\mathrm{AlO}_{x}$ layer.

\section{TDTR}

We used TDTR to measure the thermal conductivity of our r-TMD films. We used a mode-locked Ti:sapphire laser, which produced a train of pulses at a repetition rate of $74.86 \mathrm{MHz}$, with wavelength centred at $785 \mathrm{~nm}$ and a total power of $18 \mathrm{~mW}$. The steady-state temperature rise at the surface of the samples was $<4 \mathrm{~K}$ for all temperatures. For the low temperature TDTR measurements, an INSTEC stage was used with liquid nitrogen cooling; the other beam conditions were the same. The laser beam was split into pump and probe beams. A mechanical delay stage was used to delay the arrival of the probe with respect to the pump on the sample surface by changing their optical path difference, before they were focused onto the sample surface through an objective lens. The $1 / e^{2}$ radius of the focused laser beams was $10.7 \mu \mathrm{m}$. For our measurements, we modulated the pump beam at a frequency of $9.3 \mathrm{MHz}$ so that the thermoreflectance change at the sample surface could be detected by the probe beam through lock-in detection. The ratio of the in-phase and outof-phase signals from the lock-in was fitted to a thermal diffusion model. The full details of the TDTR measurement can be found elsewhere ${ }^{50,51}$.

Calculation of $\boldsymbol{\kappa}_{\perp}$. The modelling required material parameters such as heat capacity $(C)$, thickness $(h)$, interface conductance $(G)$ and thermal conductivity $(\kappa)$ for each layer. Our TDTR samples have three chemically distinct layers with the following structure (from the top): Al/r-TMD/ sapphire. In our fitting process, the heat capacities of all materials were adopted from literature ${ }^{52}$. The thickness of Al layer was obtained from picosecond acoustics using a longitudinal speed of sound of $6.42 \mathrm{~nm} \mathrm{ps}^{-1}$ (Extended Data Fig. 3e). The thickness of the r-TMD film was calculated from the product of $N$ and the interlayer spacing $(d)$. The latter was measured by performing grazing-incidence wide-angle $X$-ray spectroscopy (GIWAXS; see GIWAXS section below in the Methods and Extended Data Fig. 2) on the r-TMD films, which gave $d \approx 0.64 \mathrm{~nm}$. The total thicknesses of the $-\mathrm{MoS}_{2}$ films were $<15 \mathrm{~nm}$; thus, this layer was treated as part of the $\mathrm{Al}$-sapphire interface as a single thermal layer characterized by a single thermal conductance value $G_{1}$. We used the bulk value of the volumetric heat capacity of $1.89 \mathrm{~J} \mathrm{~K}^{-1} \mathrm{~cm}^{-3}$ for ther-MoS 2 layer. The thermal conductivity of the Al layer was calculated from the Wiedemann-Franz law using the electrical resistance of a transducer layer deposited on a bare sapphire substrate as a reference sample. The thermal conductivity of the sapphire substrate, $35 \mathrm{~W} \mathrm{~m}^{-1} \mathrm{~K}^{-1}$, was measured using the same reference sample. Thus, the only remaining free parameter to fit for was $G_{1}$. To obtain $\kappa_{\mathrm{TMD}}$ from $G_{1}$, we perform TDTR on various $N$-layer TMD films, then perform a linear fit on the effective thermal resistance $\left(R_{\mathrm{TDTR}}\right.$, equal to $\left.1 / G_{1}\right)$ versus $N$ data points; the slope of the linear fit is inversely proportional to the thermal conductivity, whereas the $y$ intercept yields the total interfacial thermal resistances $\left(R_{0}\right)$ of the top and bottom interfaces. In Extended Data Table 1, our $R_{0}$ values match the values reported in literature ${ }^{22,27,53}$. We note that, although $R_{0}$ changes depending on the chemical nature of the metal-TMD interface, the slope of the $R_{\mathrm{TDTR}}-N$ plot (which is used to extract $\kappa_{\perp}$ ) remains constant, despite the use of different transducer metals, as illustrated in Extended Data Fig. 3d.

For highly anisotropic materials, the anisotropy ratio of an in-plane thermal conductivity to a through-plane conductivity should be included in the thermal model. Despite the ultrahigh thermal anisotropy expected of our r-TMD films, our through-plane thermal conductivity measurements were probably not sensitive to the thermal conductivity anisotropy given the thinness of our $r$-TMD films. Hence, we assumed a one-dimensional thermal transport model and neglected the in-plane thermal transport in our calculations. We found that the effect of the anisotropy was significant only at a smaller modulation frequency $(f=1.12 \mathrm{MHz})$ and $1 / e^{2}$ beam radius of $-3.2 \mu \mathrm{m}$, and so we deliberately chose a larger $f$ and $1 / e^{2}$ beam radius to reduce the sensitivity of our TDTR signal to the in-plane thermal transport.

\section{Raman thermometry}

We followed a similar procedure from previous reports ${ }^{34,54,55}$ with the modification of lower pressures during measurement. All the Raman measurements were performed using a Horiba Raman spectrometer with a laser excitation wavelength of $532 \mathrm{~nm}$ and a long-working 
distance, $50 \times$ objective lens (numerical aperture $(\mathrm{NA})=0.5$ ). The $-\mathrm{MoS}_{2}$ $\mathrm{A}_{1 g}$ peak shift $(\omega)$ versus temperature $(T)$ relation was calibrated using a temperature-controlled, low-vacuum-compatible Linkam stage. For all our Raman measurements, we used the $\mathrm{A}_{1 g}$ peak since this out-of-plane vibrational mode is less sensitive to in-plane $\operatorname{strain}^{56}$. The $\omega-T$ calibration measurements were performed at atmospheric pressure and with low laser powers. The stage was purged with dry $\mathrm{N}_{2}$ gas throughout the calibration step to prevent oxidative damage to the film at high temperatures. Extended Data Fig. $5 \mathrm{~d}$ shows representative $\omega-T$ calibration curves for $N=2$ and $N=4 \mathrm{r}-\mathrm{MoS}_{2}$ films, where a linear fit was performed to obtain the temperature-dependent Raman coefficients. This process was repeated for $\mathrm{r}-\mathrm{MoS}_{2}$ films with different domain sizes $D(400 \mathrm{~nm}$ and $1 \mu \mathrm{m}$ ) for $N=1-3$ (Extended Data Fig. 5e).

To measure the in-plane thermal conductivity $\left(\kappa_{\|}\right)$of our films, the laser power $(P)$ was varied and the corresponding $\Delta \omega$ values were recorded. The in-plane thermal conductance was obtained from the reciprocal of the slope of the $\Delta \omega-P$ linear fit, which is illustrated for r- $M_{2}$ films with $N=2-5$ and $D=1 \mu \mathrm{m}$ in Fig. $3 \mathrm{c}$ and for $\mathrm{r}-\mathrm{MoS}_{2}$ films with $N=1-3$ and $D=400 \mathrm{~nm}$ in Extended Data Fig. $5 \mathrm{~b}$. As thermal conductivity changes with temperature, laser powers were kept below $250 \mu \mathrm{W}$ to induce a relatively small $\Delta T$ in the film and ensure that the value of $\kappa_{\|}$remained relatively constant. This was verified from the observation of a linear $\Delta \omega-P$ regime for $P<250 \mu \mathrm{W}$. Any higher laser powers caused the $\Delta \omega-P$ curve to deviate from the linear regime with $\frac{\mathrm{d}^{2} \omega}{\mathrm{d} P^{2}}<0$. This indicates that the local film temperature increased faster $\mathrm{d} P^{2}$ higher $P>250 \mu \mathrm{W}$, which signified that the thermal conductivity could no longer be assumed to be constant. Instead, the thermal conductivity decreased with increasing temperature, consistent with the $T$-dependent Raman measurements.

The $\Delta \omega-P$ measurements were conducted at a pressure of 15 torr to eliminate any heat loss to air. We verified that a lower pressure down to 4 mtorr gave rise to similar $\Delta \omega$ values as the measurements at 15 torr (Extended Data Fig. 5a), weighted by the beam spot size.

The other relevant input quantities for our thermal calculations were obtained as follows: the beam spot radius $\left(r_{0}\right)$ was estimated using the knife-edge method, whereby a one-dimensional Raman map was taken across a gold step edge on an Au-patterned silicon chip, and the spatial distribution of the integrated peak intensities was fitted to an error function. We measured $r_{0}=0.71 \pm 0.09 \mu \mathrm{m}$. The laser powers were measured using a Thorlabs standard silicon photodiode power sensor. The r-MoS${ }_{2}$ absorbance $A=\frac{\text { Absorbed light intensity }}{\text { Incident light intensity }}$ was measured at room temperature on a white-light microscope with a $532 \mathrm{~nm}$ band-pass filter and a low-NA condenser aperture. We measured the light intensity transmitted through and reflected from a r-MoS $\mathrm{S}_{2}$ film suspended on a TEM grid, then compared it against a blank TEM grid. The data were collected using a 12-bit SensiCam QE CCD camera. The pixel intensities were analysed using ImageJ. The values for $A$ were calculated using the formula $A=1-T-R$. We measured $A(N)$ for $N=1-5$, then fitted $A$ to a power law. $A(N)$ was found to follow the relation $A=1-0.92^{N}$ (Extended Data Fig. 5c), which matched previous reports ${ }^{20}$. We use the value $A$ measured at room temperature for our Raman analysis.

Calculation of $\boldsymbol{\kappa}_{\| \cdot}$ To obtain the value of $\boldsymbol{\kappa}_{\|}$, we used the two-dimensional thermal diffusion equation with a radial symmetry, following previous reports of Raman thermometry of two-dimensional films ${ }^{54,55}$. We assumed a Gaussian laser profile $q(r)=\frac{P A}{\left(\pi r_{0}^{2}\right) t} \quad \exp \left(-\frac{r^{2}}{r_{0}^{2}}\right)$. We solved for $\kappa_{\|}$numerically using the following equations:

$$
\begin{gathered}
\kappa_{\|} \frac{1}{r} \frac{\mathrm{d}}{\mathrm{d} r}\left(r \frac{\mathrm{d} T_{\text {susp }}(r)}{\mathrm{d} r}\right)+q(r)=0 ; r<R \\
\kappa_{\|} \frac{1}{r} \frac{\mathrm{d}}{\mathrm{d} r}\left(r \frac{\mathrm{d} T_{\text {supp }}(r)}{\mathrm{d} r}\right)-\frac{G}{t}\left[T_{\text {supp }}(r)-T_{\mathrm{a}}\right]=0 ; r>R
\end{gathered}
$$

applying the boundary conditions

$$
\begin{gathered}
T_{\text {susp }}(R)=T_{\text {supp }}(R) \\
\frac{\mathrm{d} T_{\text {susp }}}{\mathrm{d} r}(R)=\frac{\mathrm{d} T_{\text {supp }}}{\mathrm{d} r}(R)
\end{gathered}
$$

where $r$ is the distance from TEM hole centre, $P$ is the laser power, $t$ is the film thickness, $R$ is the TEM hole radius, $T$ is the film temperature where $T_{\text {susp }}, r \leq R$ and $T_{\text {supp }}, r \geq R, T_{\mathrm{a}}$ is the ambient temperature, $A$ is the fraction of laser power absorbed, and $G=10 \mathrm{MW} \mathrm{m}^{-2} \mathrm{~K}^{-1}$ is the interfacial thermal conductance between $r-\mathrm{MoS}_{2}$ and $\mathrm{SiN}_{x}$.

We solved for the expression of $T(r)$ and obtained an expression for the average temperature measured by the Raman shift

$$
T_{\mathrm{m}}=\frac{\int_{0}^{R} T(r) r \times \exp \left(-\frac{r^{2}}{r_{0}^{2}}\right) \mathrm{d} r}{\int_{0}^{R} r \times \exp \left(-\frac{r^{2}}{r_{0}^{2}}\right) \mathrm{d} r}
$$

$\kappa_{\|}$was obtained by substituting the experimentally measured value for $T_{\mathrm{m}}$ and solving the above equation numerically for $\kappa_{\| \cdot}$. We calculated $\kappa_{\|}$ for each $N$, and we reported the average value in the main text.

The total measurement uncertainty reported in the main text was calculated based on the error assessment for individual parameters. We used an approximate analytical solution

$$
\begin{gathered}
\Delta T=T_{\mathrm{m}}-T_{\mathrm{a}} \approx-\left(\frac{P_{\mathrm{abs}}}{\kappa_{\|}}\right)\left(\frac{1}{2 \pi r t}\right) \ln \left(\frac{R}{r_{0}}\right) \\
\kappa_{\|} \approx\left(\left(\frac{\Delta \omega}{\Delta P}\right)^{-1}\left(\frac{\Delta \omega}{\Delta T}\right)\right)\left(\frac{A_{0}}{2 \pi d}\right) \ln \left(\frac{R}{r_{0}}\right)
\end{gathered}
$$

where $\omega$ is the Raman frequency of $\mathrm{A}_{1 g}$ peak, $A_{0}$ is the absorption of the monolayer, and $d$ is the thickness of a monolayer. The difference between the full numerical solution and this analytical form is below $3 \%$. We identified the following independent quantities that carry uncertainty for consideration in our overall uncertainty estimation of $\kappa_{\| \cdot}$. 1. $\frac{\Delta P}{\Delta T}=\left(\frac{\Delta \omega}{\Delta P}\right)^{-1}\left(\frac{\Delta \omega}{\Delta T}\right):$ the associated uncertainty was derived from the error in the linear fit of $\left(\frac{\Delta \omega}{\Delta P}\right)$ and $\left(\frac{\Delta \omega}{\Delta T}\right)$ for every sample measured. The total uncertainty in the average $\frac{\Delta P}{\Delta T}$ value was $9 \%$ for $D=1 \mu \mathrm{m}$ and $8 \%$ for $D=400 \mathrm{~nm}$.

2. $\left(\frac{A_{0}}{2 \pi d_{0}}\right)$ : the uncertainty in $A_{0}$ from the $A(N)$ fit was $4 \%$.

3. $\ln \left(\frac{R}{r_{0}}\right)$ : the uncertainty originated from the uncertainty in $r_{0}$. From 14 repeated measurements of the beam spot size using the knife-edge method, we calculated the standard deviation of $r_{0}$ to be $12 \%$, which translated to an uncertainty in the expression $\ln \left(\frac{R}{r_{0}}\right)$ to be $9 \%$. Total uncertainty in $\kappa_{\|}: 13 \%$ (for both $D=1 \mu \mathrm{m}$ and $D=400 \mathrm{~nm}$ ).

Variable pressure Raman thermometry measurements. Previous Raman thermometry measurements on graphene films ${ }^{57}$ and carbon nanotubes ${ }^{58}$ had shown an appreciable difference between measurements performed in air and at lower pressures, as well as in different gaseous environments. We extended the same precaution and repeated our Raman thermometry measurements at low pressures to reduce heat dissipation to air, an extra heat loss channel that would lead to an overestimation of the thermal conductivity of the r-MoS films.

Our $\Delta \omega-P$ measurements in Fig. 3 were conducted at a pressure of 15 torr. In Extended Data Fig. 5a, we compared the $\Delta \omega$ values of $N=2$ $\mathrm{r}-\mathrm{MoS}_{2}$ at three different $P(1 \mathrm{~atm}, 15$ torr and 4 mtorr $)$, after correcting for the different laser spot sizes. 
Temperature-dependent Raman thermometry for $\boldsymbol{\kappa}_{\|}$. We performed Raman thermometry while varying the ambient temperature $T_{\mathrm{a}}$ using a Linkam stage. No oxidation or sample damage was detected for any of the temperatures used. We performed the same $\Delta \omega-P$ measurements and calculated the $\kappa_{\|}$value of for each $T_{\mathrm{a}}$. We plot a $\kappa_{\|}$versus $T$ curve, where the $x$ axis is $T=T_{\mathrm{a}}$ (Extended Data Fig. 6a).

We note that the measured values of $\kappa_{\|}$here were lower than the room temperature values reported in the main text. We ascribe this to the sub-optimal growth conditions for the constituent monolayers used for this sample.

\section{r-MoS ${ }_{2}$ heat spreader experiments (electromigration of $\mathrm{Au}$ nanoelectrodes)}

All Au electrodes were fabricated on $\mathrm{Si}$ substrates with $50 \mathrm{~nm}$ dry $\mathrm{SiO}_{2}$ in three nanopatterning and deposition layers: (A) the nanoelectrodes (10 $\mu \mathrm{m}$ long, $100 \mathrm{~nm}$ wide, $15 \mathrm{~nm}$ thick);(B) the contact pads that would interface with the external electronics $(200 \mu \mathrm{m}$ long, $300 \mu \mathrm{m}$ wide, $100 \mathrm{~nm}$ thick); and (C) the leads connecting the nanoelectrodes and the contact pads $(\sim 1,000 \mu \mathrm{m}$ long, $50 \mu \mathrm{m}$ wide, $15 \mathrm{~nm}$ thick $)$.

We first defined the leads (B) and then the contact pads (C) using standard photolithography, and electron-beam evaporation of $\mathrm{Ti}$ $(1 \mathrm{~nm}) /$ Au and lift-off. The final step was defining the nanoelectrodes (A) using electron-beam lithography, deposition of $15 \mathrm{~nm} \mathrm{Au}$, and lift-off.

Electron-beam lithography. We used a bilayer of resists: copolymer $\mathrm{P}(\mathrm{MMA}-\mathrm{MAA} 11 \%)$ in ethyl lactate and $950 \mathrm{~K}$ PMMA A4. The writing was executed with a Raith EBPG 5000 Plus E-beam writer with the beam conditions of $25 \mathrm{nA}$ current, dose of $1,200 \mu \mathrm{C} \mathrm{cm}^{-2}, 300 \mu \mathrm{m}$ aperture size, $100 \mathrm{kV}$ accelerating voltage.

Film transfer. After the nanoelectrodes, leads and pads were fabricated, the device was cleaned with an $\mathrm{O}_{2}$ plasma for 30 s to remove any resist residue and to promote adhesion of the r- $\mathrm{MoS}_{2}$ film to the Au electrodes and the $\mathrm{SiO}_{2}$ surface. A PMMA coated $N=16 \mathrm{r}-\mathrm{MoS}_{2}$ film was transferred onto the electrodes using the same process as the stacking method as outlined above. The PMMA on the r-MoS $\mathrm{S}_{2}$ film was removed by immersing the entire chip in toluene at $60^{\circ} \mathrm{C}$ for $1 \mathrm{~h}$.

Electrical measurements. All measurements were performed in ambient conditions with a home-built probe station in a two-probe geometry. To measure $I_{c}$ in Fig. $4 \mathrm{~d}$, we swept the voltage bias in only one direction at a rate such that the rate in current increase is $0.05 \mathrm{~mA}$ per $20 \mathrm{~s}$.

For comparison, we deposited $\mathrm{SiN}_{x}$ onto Au electrodes $(10 \mu \mathrm{m}$ long, $10 \mathrm{~nm}$ thick, $100 \mathrm{~nm}$ wide) using plasma-enhanced chemical vapour deposition with the following conditions: 10 s deposition at $90^{\circ} \mathrm{C}$ and 10 torr and 1,000 W plasma power, with $25 \mathrm{SCCM} / 35 \mathrm{SCCM} \mathrm{SiH}_{4}$ and $\mathrm{N}_{2}$ as the precursors. The film thickness was measured via ellipsometry to be $16 \mathrm{~nm}$.

\section{Computational methodology}

Structural models. Structural models were created according to an algorithm previously described in literature ${ }^{59}$, which was implemented in Python using the atomic simulation environment package ${ }^{60}$. The structure models were subsequently relaxed using an analytic bond-order potential ${ }^{61}$ and implemented in the LAMMPS package ${ }^{62}$.

The main $\mathrm{r}-\mathrm{MoS}_{2}$ model used in the simulations described here comprised 10 randomly stacked layers with a total of 10,152 atoms. The 10 layers came in pairs; each pair was related by a $60^{\circ}$ rotation. The four primitive angles present in the stack are 16.1, 25.28, 34.72 and $43.9^{\circ}$. Due to strain, each layer contained a different number of atoms in accordance with strains of around $10 \%$.

The bulk structure used in the MD simulations comprised 40 layers ( 20 conventional unit-cells) with a total of 26,880 atoms and cell vectors of $44.44,43.98$ and $243.57 \AA$.
HNEMD simulations. The interatomic potential in our simulations produces the expected slight increase in the interlayer spacing in $\mathrm{r}-\mathrm{MoS}_{2}$ and yields thermal conductivities and phonon dispersions of bulk $\mathrm{MoS}_{2}$ that agree with previous experimental observations and Boltzmann transport calculations based on density functional theory ${ }^{38}$, confirming our MD model's suitability for this study. The structures described above were driven by an optimized driving force (Extended Data Fig. 8a) and subsequently relaxed, after which the thermal conductivity was computed using HNEMD simulations ${ }^{63}$ and implemented in the graphics processing units molecular dynamics (GPUMD) package ${ }^{29}$. We also included the effects of thermal expansion in the simulations (Extended Data Fig. 8b). The calculated $\kappa_{\perp}$ values of $r-\mathrm{MoS}_{2}$ are higher than the experimental values. We attribute the discrepancy to our neglecting any quantum effects and all boundary scattering in our simulations. Including such effects could further improve results from simulations. Statistics and averages were gathered from ten independent simulation runs for each system and temperature. The other parameters used in these simulations are compiled in Extended Data Table 2.

Phonon dispersion and lifetimes. We first generated the bulk $\mathrm{MoS}_{2}$ phonon dispersion in the harmonic limit as a reference to phonon dispersion calculations using MD simulations. We computed the harmonic $(0 \mathrm{~K})$ phonon dispersion using the PHONOPY package ${ }^{64}$. Forces were computed for $6 \times 6 \times 2$ supercells using the LAMMPS code. Lifetimes were calculated using the lowest applicable order of perturbation theory using the PHONO3PY package ${ }^{65}$, which also provided us with the thermal conductivity as obtained from a direct solution of the Boltzmann transport equation ${ }^{66}$. In these calculations, the Brillouin zone was sampled using a $10 \times 10 \times 10$ Г-centred q-point mesh, which was chosen for consistency with the supercell used in the HNEMD simulations.

Next, we compared the dispersion of bulk $\mathrm{MoS}_{2}$ to that calculated using MD simulations to verify the accuracy of our MD simulations for calculating the phonon dispersion of $\mathrm{r}-\mathrm{MoS}_{2}$. For both bulk and $\mathrm{r}-\mathrm{MoS}_{2}$, we extracted the phonon dispersions and lifetimes at $300 \mathrm{~K}$ by analysing the longitudinal and transverse current correlation functions generated by MD simulations in the microcanonical (NVE) ensemble using dynasor ${ }^{67}$. The MD simulations details were otherwise identical to the HNEMD simulations. The obtained correlation functions were Fourier transformed and fitted to peak shape functions corresponding to (over)damped harmonic oscillators using the full expressions given in the dynasor paper ${ }^{67}$ to obtain phonon frequencies and lifetimes.

Finite-element analysis. We used the COMSOL software to simulate the steady-state temperature distribution in a Au electrode on $\mathrm{SiO}_{2} /$ Si substrate. Our geometry contains an Au electrode that is $100 \mathrm{~nm}$ wide, $15 \mathrm{~nm}$ thick, and $10 \mu \mathrm{m}$ long on a $50-\mathrm{nm}$-thick $\mathrm{SiO}_{2}$ layer on a $\mathrm{Si}$ substrate. We layer a 10 -nm-thick $\mathrm{MoS}_{2}$ film onto the Au electrode and the $\mathrm{SiO}_{2}$ layer. For the thermal anisotropy consideration, we define the thermal conductivity slow axis direction to always be perpendicular to the film's bottom surface in contact with the substrate or the $\mathrm{Au}$ electrode, including the Au electrode side walls.

We supply the Au electrode with $8 \mathrm{~mW}$ uniformly over the entire volume as the heat source, matching the power conditions at which the Au electrode fails in our experiments. As the boundary condition, we set the bottom surface on the Si substrate to be at $293.15 \mathrm{~K}$. We also account for all the interfacial thermal resistances between heterogeneous surfaces in our calculations, which include $\mathrm{r}-\mathrm{MoS}_{2} / \mathrm{Au}, \mathrm{r}-\mathrm{MoS} / \mathrm{SiO}_{2}$, $\mathrm{Au} / \mathrm{SiO}_{2}, \mathrm{SiO}_{2} / \mathrm{Si}\left(\right.$ refs. $\left.{ }^{68-71}\right)$. All effects of radiation are neglected as they do not affect the temperature values in our simulations.

\section{Low-frequency Raman measurements}

The low-frequency Raman spectra of $N=2,3$ and $4 \mathrm{r}-\mathrm{MoS}_{2}$ films, along with the spectrum for $\mathrm{MoS}_{2}$, are shown in Extended Data Fig. 4a. From the layer-dependence of the peak positions, we assigned these to be 
the breathing modes of $\mathrm{MoS}_{2}\left(\right.$ ref. $\left.^{72}\right)$. We did not observe any peaks corresponding to shear modes in $\mathrm{r}-\mathrm{MoS}_{2}$. Our findings agree with theoretical studies of low-frequency Raman modes of twisted $\mathrm{MoS}_{2}$ bilayers, which showed that the shear mode peaks redshift to below the detection capabilities $\left(2 \mathrm{~cm}^{-1}\right)^{73}$. The positions of the breathing mode peaks of the $\mathrm{r}-\mathrm{MoS}_{2}$ films were close to those in exfoliated few layer $\mathrm{MoS}_{2}$ (Extended Data Fig. 4b) as reported in literature ${ }^{72,74}$. This observation agreed with our MD simulations that suggested that the transverse vibrational mode was suppressed by interlayer rotation, while the longitudinal vibrational mode was retained.

\section{GIWAXS}

The GIWAXS measurement was performed using SAXSLAB (XENOCS)'s

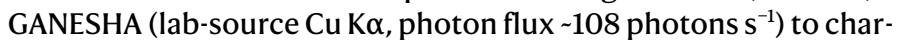
acterize the interlayer spacing of $r-\mathrm{MoS}_{2}$ films. An $N=10 \mathrm{r}-\mathrm{MoS}_{2}$ film was prepared on $\mathrm{SiO}_{2} / \mathrm{Si}$ substrate. The incidence angle of the X-ray beam was $0.2^{\circ}$ and the integration time was $\sim 60 \mathrm{~s}$. Radially integrating the two-dimensional diffraction images along the out-of-plane direction produced the diffraction spectrum along the $c$ axis shown in Extended Data Fig. 2.

The peak position of $14^{\circ}$ corresponded to an interlayer spacing of $6.4 \AA$ in the [0 01] direction, which matched previous reports of r-TMD films ${ }^{20}$.

\section{Data availability}

The data that support the findings of this study are available from the corresponding authors on reasonable request. Source data are provided with this paper.

\section{Code availability}

All code used in this work is available from the corresponding authors on reasonable request.

49. Cueva, P., Hovden, R., Mundy, J. A., Xin, H. L. \& Muller, D. A. Data processing for atomic resolution electron energy loss spectroscopy. Microsc. Microanal. 18, 667-675 (2012).

50. Cahill, D. G. Analysis of heat flow in layered structures for time-domain thermoreflectance. Rev. Sci. Instrum. 75, 5119-5122 (2004).

51. Liu, J. et al. Simultaneous measurement of thermal conductivity and heat capacity of bulk and thin film materials using frequency-dependent transient thermoreflectance method. Rev. Sci. Instrum. 84, 034902 (2013).

52. Ditmars, D. A., Plint, C. A. \& Shukla, R. C. Aluminum. I. Measurement of the relative enthalpy from 273 to $929 \mathrm{~K}$ and derivation of thermodynamic functions for $\mathrm{Al}(\mathrm{s})$ from O K to its melting point. Int. J. Thermophys. 6, 499-515 (1985).

53. Yue, X. F. et al. Measurement of interfacial thermal conductance of few-layer $\mathrm{MoS}_{2}$ supported on different substrates using Raman spectroscopy. J. Appl. Phys. 127, 104301 (2020).

54. Lee, J.-U., Yoon, D., Kim, H., Lee, S. W. \& Cheong, H. Thermal conductivity of suspended pristine graphene measured by Raman spectroscopy. Phys. Rev. B 83, 081419 (2011)

55. Cai, W. et al. Thermal transport in suspended and supported monolayer graphene grown by chemical vapor deposition. Nano Lett. 10, 1645-1651 (2010).

56. Wang, Y., Cong, C., Qu, C. \& Yu, T. Raman spectroscopy study of lattice vibration and crystallographic orientation of monolayer $\mathrm{MoS}_{2}$ under uniaxial strain. Small 9, 2857-2861 (2013).

57. Chen, S. et al. Raman measurements of thermal transport in suspended monolayer graphene of variable sizes in vacuum and gaseous environments. ACS Nano 5, 321-328 (2011).

58. Hsu, I.-K., Pettes, M. T., Aykol, M., Shi, L. \& Cronin, S. B. The effect of gas environment on electrical heating in suspended carbon nanotubes. J. Appl. Phys. 108, 084307 (2010).

59. Hermann, K. Periodic overlayers and moiré patterns: theoretical studies of geometric properties. J. Phys. Condens. Matter 24, 314210 (2012).

60. Hjorth Larsen, A. et al. The atomic simulation environment-a Python library for working with atoms. J. Phys. Condens. Matter 29, 273002 (2017).
61. Liang, T., Phillpot, S. R. \& Sinnott, S. B. Parametrization of a reactive many-body potential for Mo-S systems. Phys. Rev. B 79, 245110 (2009).

62. Plimpton, S. Fast parallel algorithms for short-range molecular dynamics. J. Comput. Phys. 117, 1-19 (1995).

63. Evans, D. J. Homogeneous NEMD algorithm for thermal conductivity-application of non-canonical linear response theory. Phys. Lett. A 91, 457-460 (1982).

64. Togo, A. \& Tanaka, I. First principles phonon calculations in materials science. Scr. Mater. 108, 1-5 (2015).

65. Togo, A., Chaput, L. \& Tanaka, I. Distributions of phonon lifetimes in Brillouin zones. Phys. Rev. B 91, 094306 (2015).

66. Chaput, L. Direct solution to the linearized phonon Boltzmann equation. Phys. Rev. Lett. 110, 265506 (2013).

67. Fransson, E., Slabanja, M., Erhart, P. \& Wahnström, G. DYNASOR-a tool for extracting dynamical structure factors and current correlation functions from molecular dynamics simulations. Adv. Theory Simul. 4, 2000240 (2021).

68. Yalon, E. et al. Energy dissipation in monolayer $\mathrm{MoS}_{2}$ electronics. Nano Lett. 17, 3429-3433 (2017).

69. Freedy, K. M., Olson, D. H., Hopkins, P. E. \& McDonnell, S. J. Titanium contacts to $\mathrm{MoS}_{2}$ with interfacial oxide: interface chemistry and thermal transport. Phys. Rev. Mater. 3, 104001 (2019)

70. Lombard, J., Detcheverry, F. \& Merabia, S. Influence of the electron-phonon interfacial conductance on the thermal transport at metal/dielectric interfaces. J. Phys. Condens. Matter 27, 015007 (2015).

71. Kimling, J., Philippi-Kobs, A., Jacobsohn, J., Oepen, H. P. \& Cahill, D. G. Thermal conductance of interfaces with amorphous $\mathrm{SiO}_{2}$ measured by time-resolved magneto-optic Kerr-effect thermometry. Phys. Rev. B 95, 184305 (2017).

72. Zhao, Y. et al. Interlayer breathing and shear modes in few-trilayer $\mathrm{MoS}_{2}$ and $\mathrm{WSe}_{2}$. Nano Lett. 13, 1007-1015 (2013).

73. Huang, S. et al. Low-frequency interlayer Raman modes to probe interface of twisted bilayer $\mathrm{MoS}_{2}$. Nano Lett. 16, 1435-1444 (2016).

74. Zhang, X. et al. Raman spectroscopy of shear and layer breathing modes in multilayer MoS 2 . Phys. Rev. B 87, 115413 (2013).

Acknowledgements We thank D. F. Ogletree, J. Jureller, A. J. Mannix, J.-U. Lee, K.-H. Lee and M. Lee for their helpful discussions. We also acknowledge J.-H. Kang, A. Ye and C. Liang for their help with materials preparation. Primary funding for this work comes from Air Force Office of Scientific Research MURI projects (FA9550-18-1-0480 and FA9550-16-1-0031). Material growths done by F.M. and C.P. are partially supported by the University of Chicago MRSEC (NSF DMR-2011854) and Samsung Advanced Institute of Technology. This work makes use of the characterization facilities of the University of Chicago MRSEC (NSF DMR-2011854) and the Pritzker Nanofabrication Facility at the University of Chicago, which receives support from SHyNE Resource (NSF ECCS-1542205), a node of NSF's NNCI network. TDTR measurements are supported by Office of Naval Research MURI grant N00014-16-1-2436 and are carried out in Frederick Seitz Materials Research Laboratory at the University of Illinois at

Urbana-Champaign. F.E., E.F. and P.E. are funded by the Knut and Alice Wallenberg Foundation (2014.0226), the Swedish Research Council (2015-04153 and 2018-06482), and the FLAG-ERA JTC-2017 project MECHANIC funded by the Swedish Research Council (VR 2017-06819); they acknowledge the computer time allocations by the Swedish National Infrastructure for Computing at NSC (Linköping) and C3SE (Gothenburg). A.R. and electron microscopy at the Cornell Center for Materials Research are supported by the NSF MRSEC grant DMR-1719875. The Titan microscope was acquired with the NSF MRI grant DMR-1429155. F.M. acknowledges support by the NSF Graduate Research Fellowship Program under grant no. DGE-1746045. Y.Z. acknowledges support by the Camille and Henry Dreyfus Foundation, Inc., under the Dreyfus Environmental Postdoc award EP-16-094.

Author contributions S.E.K., J.S., and J.P. conceived the experiments. S.E.K. produced and performed the structural characterizations of the r-TMD films, and ran the finite-element simulations. F.M. and C.P. grew the TMD monolayers. A. Rai and D.G.C. performed the TDTR measurements. F.E., E.F. and P.E. conducted the atomistic simulations. P.P. fabricated the Au electrodes for the electromigration experiments. A. Ray and D.A.M. performed the STEM imaging. S.E.K. performed the Raman measurements and analysed the data with the help of D.G.C. and Y.Z. S.E.K., F.M. and J.P. wrote the manuscript. J.P., D.G.C. and P.E. oversaw the project. All authors discussed the manuscript and provided feedback.

Competing interests The authors declare no competing interests.

\section{Additional information}

Supplementary information The online version contains supplementary material available at https://doi.org/10.1038/s41586-021-03867-8.

Correspondence and requests for materials should be addressed to Paul Erhart, David G. Cahill or Jiwoong Park.

Peer review information Nature thanks Davide Donadio, M. Muñoz Rojo and Mariusz Zdrojek for their contribution to the peer review of this work.

Reprints and permissions information is available at http://www.nature.com/reprints. 


\section{Article}

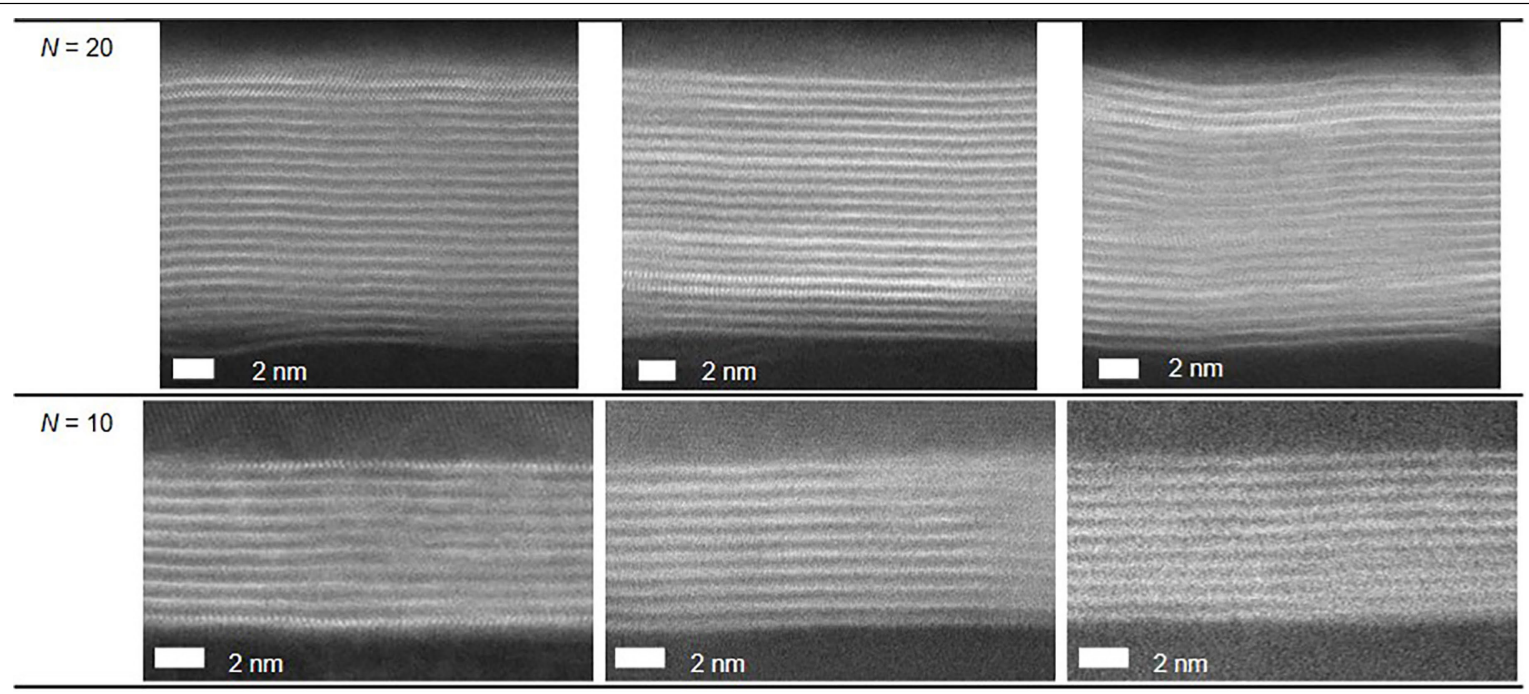

Extended Data Fig. 1 |Cross-sectional TEM images of $\boldsymbol{N}=\mathbf{2 0}$ and $10 \mathrm{r}-\mathbf{M o S}_{2}$ on $\mathbf{A l O}_{x}$. Each set of $N$ images are taken from the same sample at different locations The $N=10$ films are coated with Al that was electron beam evaporated onto the surface. 


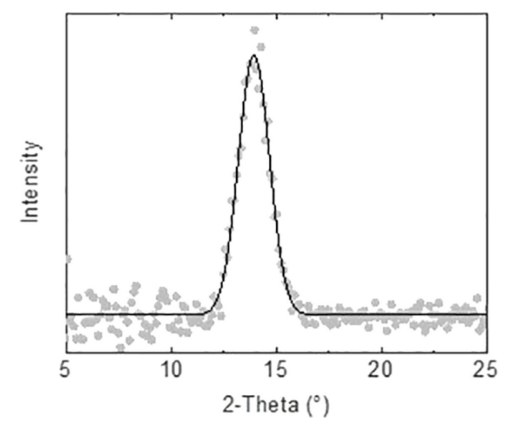

Extended Data Fig. 2 |GIWAXS data of $N=10 \mathrm{r}-\mathrm{MoS}_{2}$. The peak position corresponds to a $2 \theta$ value of $14^{\circ}$, which translates to an interlayer spacing of $6.4 \AA$ (scattering direction). 


\section{Article}
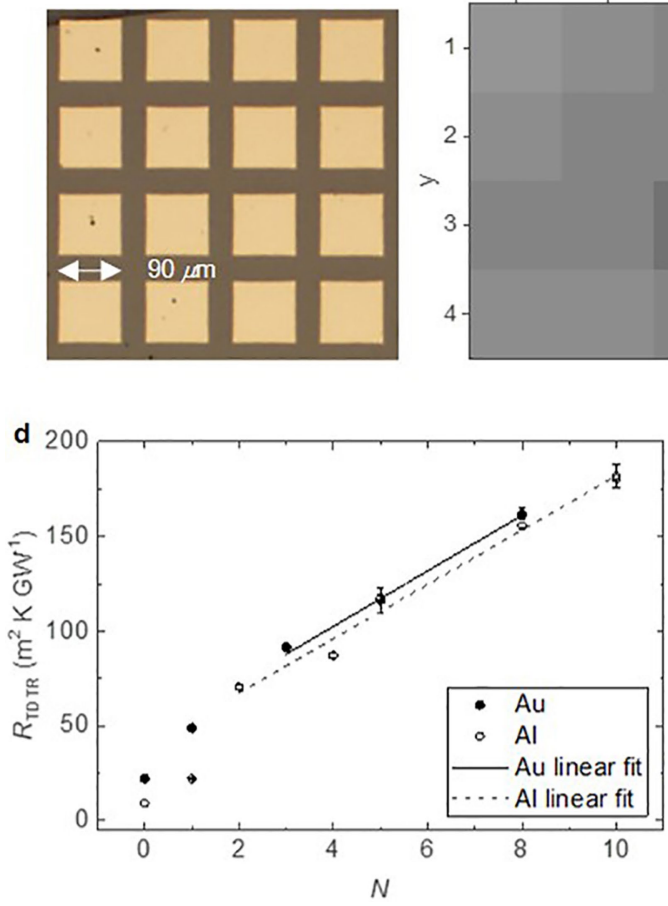

Extended Data Fig. 3 | Additional TDTR measurements and details.

a, Microscope image of an $N=10 \mathrm{r}-\mathrm{MoS}_{2}$ film coated with a square grid of Al pads. b, $4 \times 4$ TDTR map of $R_{\mathrm{TDTR}}$ of an $N=10 \mathrm{r}-\mathrm{MoS}_{2}$ film. c, Histogram of $R_{\mathrm{TDTR}}$ array measurements. d, TDTR measurements of $N \leq 10 \mathrm{r}$-TMD films coated with Au or Al. The error bars denote s.d.; number of TDTR measurements per film
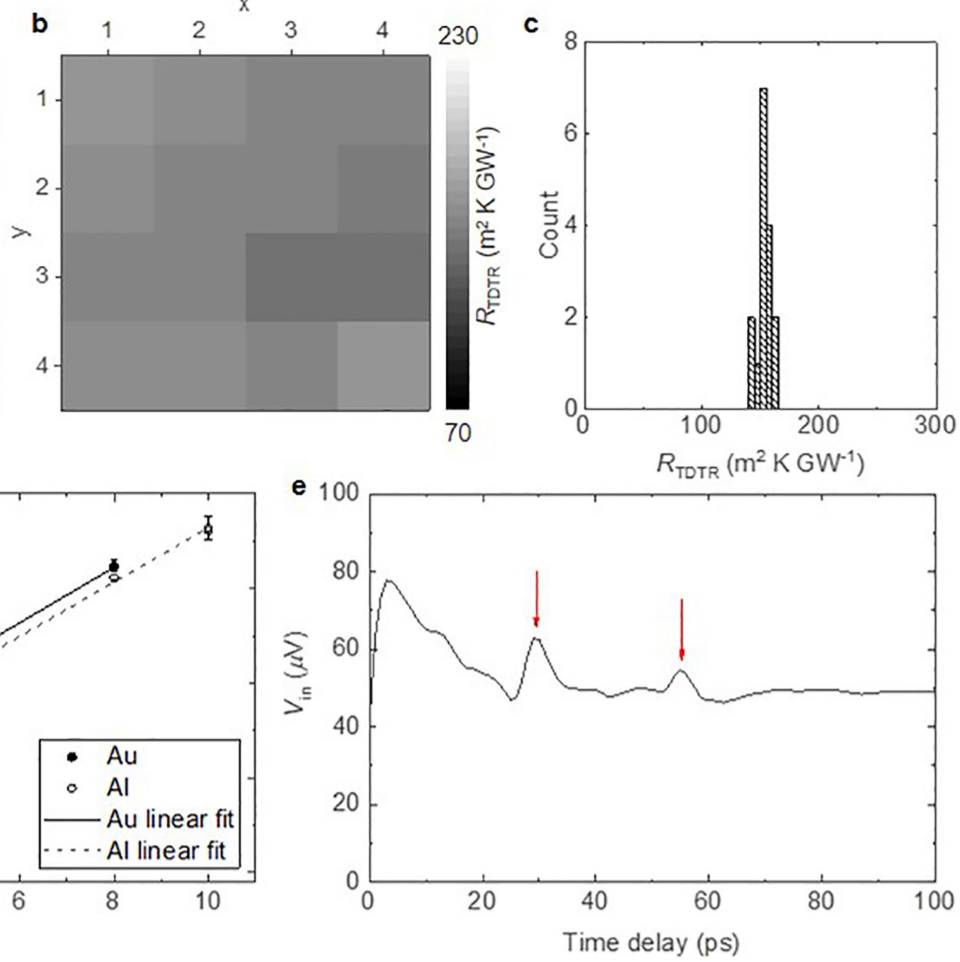

sample, $n=3$ for Au samples; $n=3-5$ for the Al samples. e, Picosecond acoustics of a $\mathrm{MoS}_{2}$ monolayer on thick sapphire substrate, coated with an Al transducer layer. The $y$ axis $V_{\text {in }}$ is the in-phase signal of the lock-in amplifier. The red arrows indicate the acoustic waves reflected at the $\mathrm{Al} / \mathrm{MoS}_{2}$ interface. 


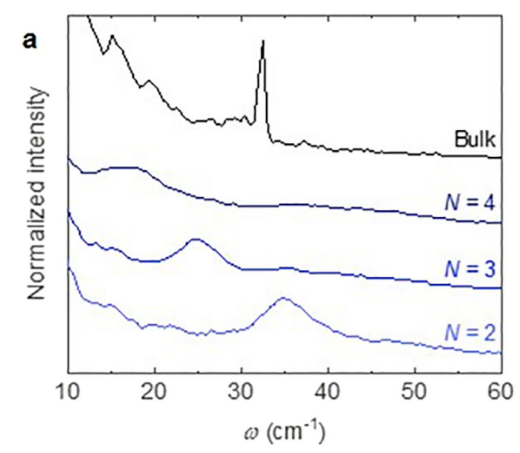

Extended Data Fig. 4 | Low-frequency Raman modes of r-MoS ${ }_{2}$. a, Raman spectra reflecting the breathing modes (BM) of $\mathrm{r}-\mathrm{MoS}_{2}$ (blue) and the shear mode (SM) for $\mathrm{MoS}_{2}$. b, The low-frequency Raman peak positions of r-MoS${ }_{2}$ and

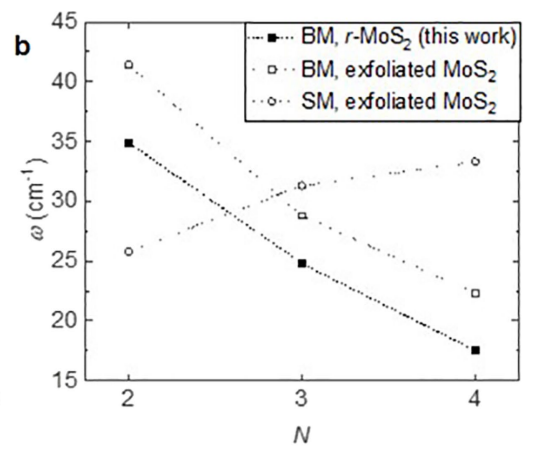

exfoliated $\mathrm{MoS}_{2}$. The filled squares indicate the BM peak positions of r-MoS . The open squares indicate the BM peak positions of exfoliated $\mathrm{MoS}_{2}$, and the open circles indicate the SM peak positions of exfoliated $\mathrm{MoS}_{2}{ }^{72}$. 

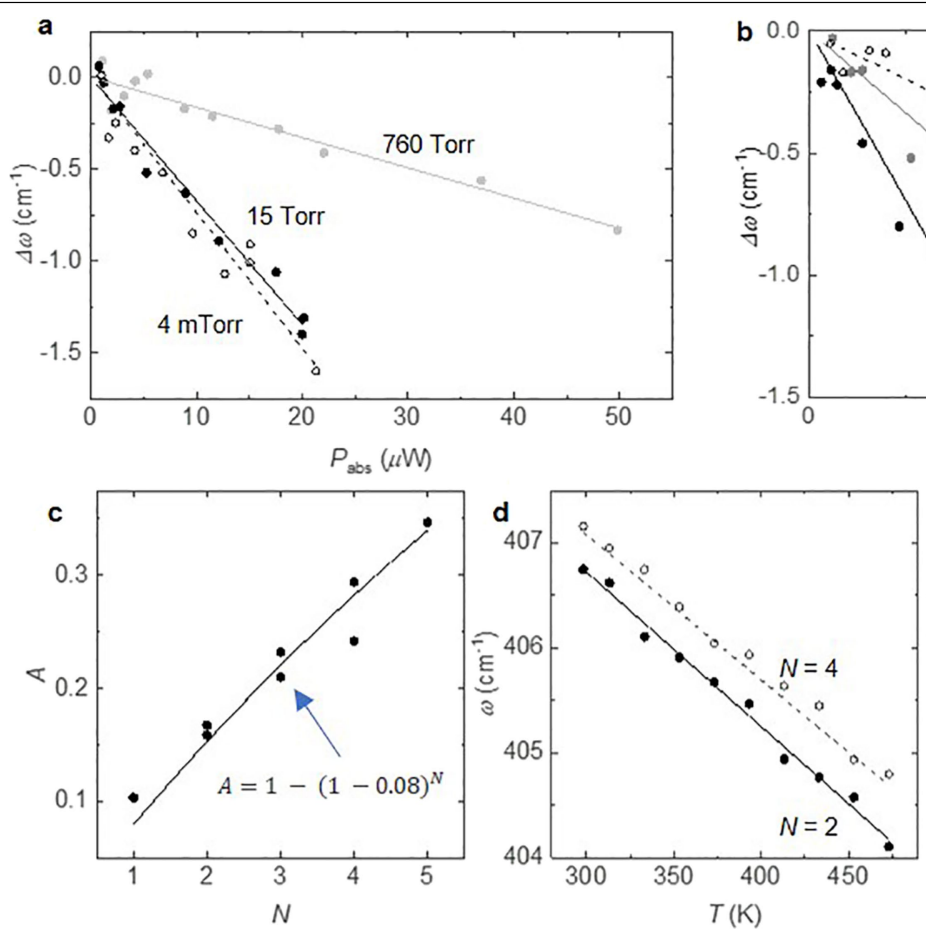
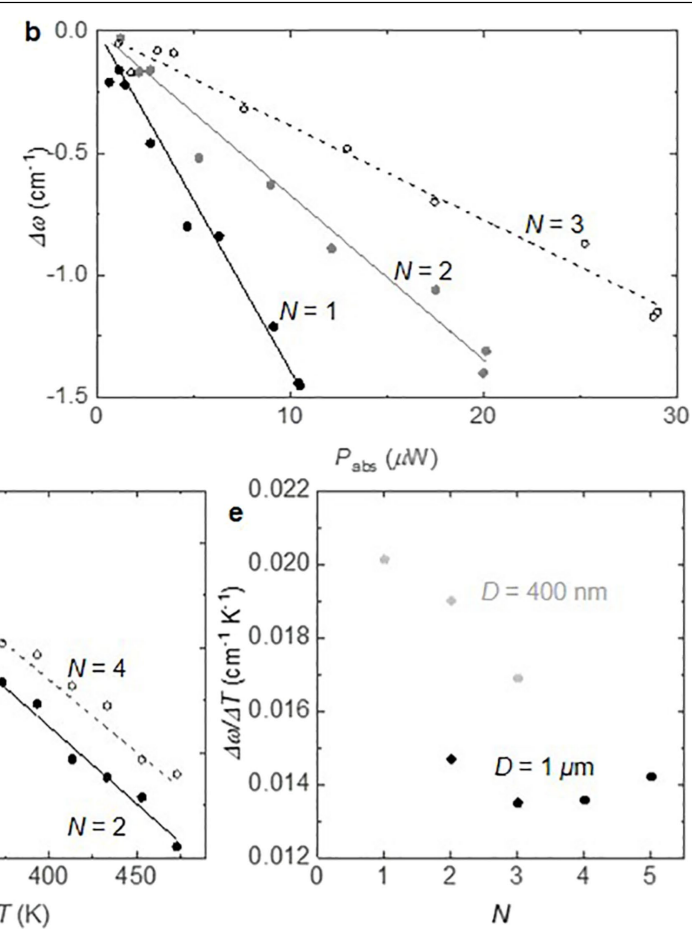

Extended Data Fig. $5 \mid$ Raman thermometry on r-MoS $\mathbf{S}_{2}$ films. a, $\Delta \omega-P_{\text {abs }}$ curves of representative $N=2 \mathrm{r}-\mathrm{MoS}_{2}$ films at different pressures. The $P_{\text {abs }}$ values along the $x$ axis are normalized to account for the slight differences in beam spot sizes $(\Delta r=20 \%$ ). The results at 15 torr and 4 mtorr signify no effect of reducing the pressure to below 15 torr. $\Delta \omega$ was approximately five-fold smaller at atmospheric pressure due to the extra heat loss channel by air. b, $\Delta \omega-P_{\text {abs }}$ curves of r-MoS${ }_{2}$ films made up of $D=400 \mathrm{~nm}$ (grain size) monolayers. c, Optical absorption of suspended $\mathrm{r}-\mathrm{MoS}_{2}$ films, which follows the trend $A=1-\left(1-A_{0}\right)^{N}$, where $A_{0}$ is the monolayer absorptance. From the fit, $A_{0}=0.08 \pm 0.003$. d, $\omega-T$ calibration measurements of suspended $\mathrm{r}-\mathrm{MoS}_{2}$ films $(D=1 \mu \mathrm{m})$, with the $N=2$ and $N=4$ data as the representative curves. e, $\omega-T$ slopes versus layer number for all films, with $D=400 \mathrm{~nm}$ or $1 \mu \mathrm{m}$. 


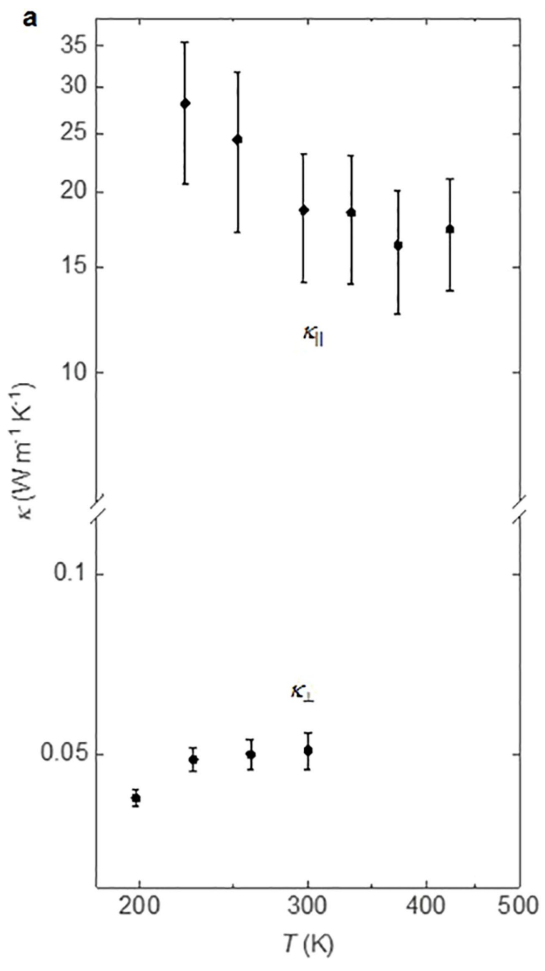

Extended Data Fig. $6 \mid \boldsymbol{\kappa}(T)$ and $\rho$ of r-MoS ${ }_{2} \cdot \mathbf{a}, \kappa(T)$ of r-MoS ${ }_{2}$, with $\kappa_{\|}$measured using Raman thermometry of $N=4 \mathrm{r}-\mathrm{MoS}_{2}$, and $\kappa_{\perp}$ measured via TDTR as reported in Fig. 2c. The error bars are the propagated uncertainties from the calculation of the conductance value for each $N$. The error bars denote the propagated uncertainty of the calculations from the input parameters. We observed that $\boldsymbol{K}_{\|}$decreased with $T$, alluding to phonon-mediated heat transport

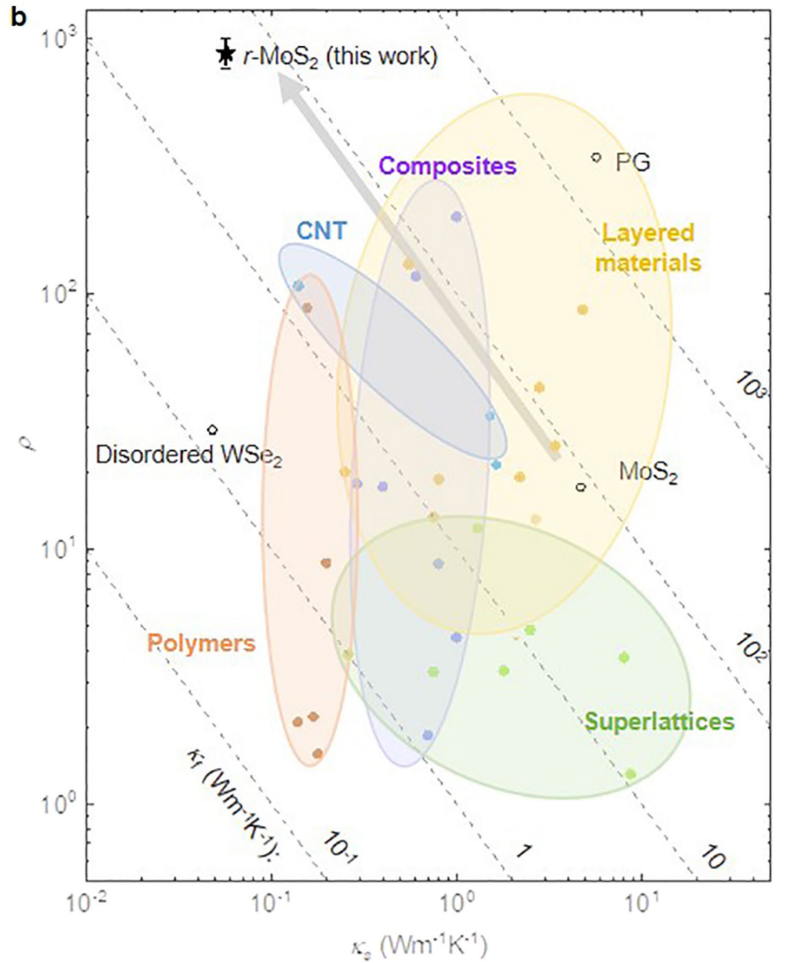

and attesting to the long-range crystallinity of the $-\mathrm{MoS}_{2}$ films in-plane. This was in contrast with the $\kappa_{\perp}(T)$ behaviour (Fig. $2 \mathrm{C}$ ), which showed a slightly increasing trend. $\mathbf{b}$, Catalogue of experimentally measured anisotropy ratios at room temperature versus slow-axis thermal conductivity $\left(\kappa_{\mathrm{s}}\right)$ of thermally anisotropic materials from literature, by category. 
Article
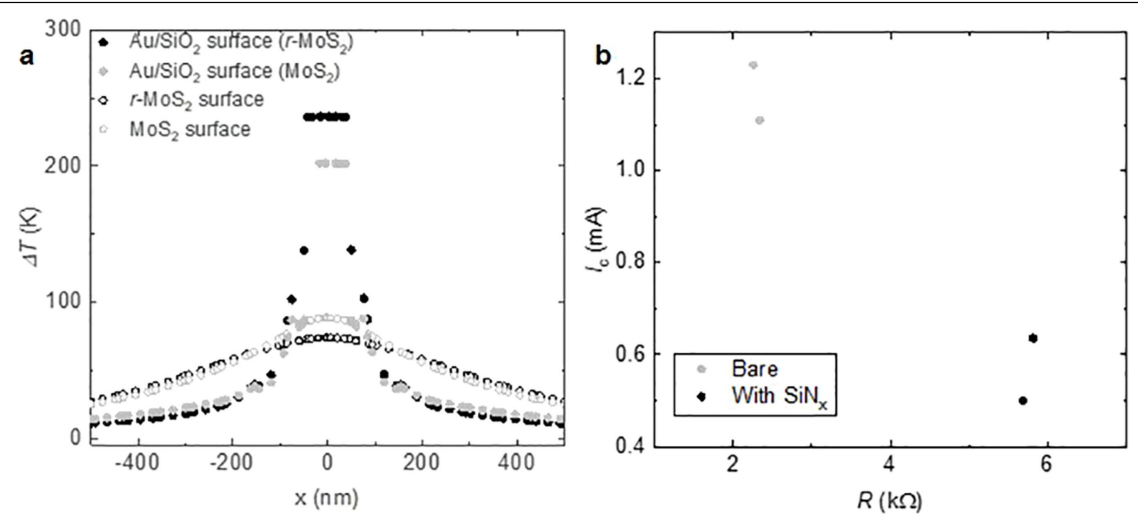

Extended Data Fig. $7 \mid$ r-MoS, efficacy as a heat spreader. a, Finite element simulations of the linear temperature profiles of Au electrodes covered with $\mathrm{MoS}_{2}$ and $\mathrm{r}-\mathrm{MoS}_{2} \cdot \mathbf{b}, \mathrm{SiN}_{x}$ as heat spreaders for Au electrodes. Electrical properties of 10-nm-thick, 100 -nm-wide and $10-\mu \mathrm{m}$-long Au electrodes before and after $16 \mathrm{~nm} \mathrm{SiN}_{x}$ film deposition onto the electrodes using plasma-enhanced chemical vapour deposition. In contrast to $\mathrm{r}-\mathrm{MoS}_{2}$, the direct deposition of an ultrathin inorganic film such as $\mathrm{SiN}_{x}$ with a comparable $\kappa$ to $\kappa_{\|}$ of $\mathrm{r}-\mathrm{MoS}_{2}$ negatively affects the performance of the Au electrodes. 

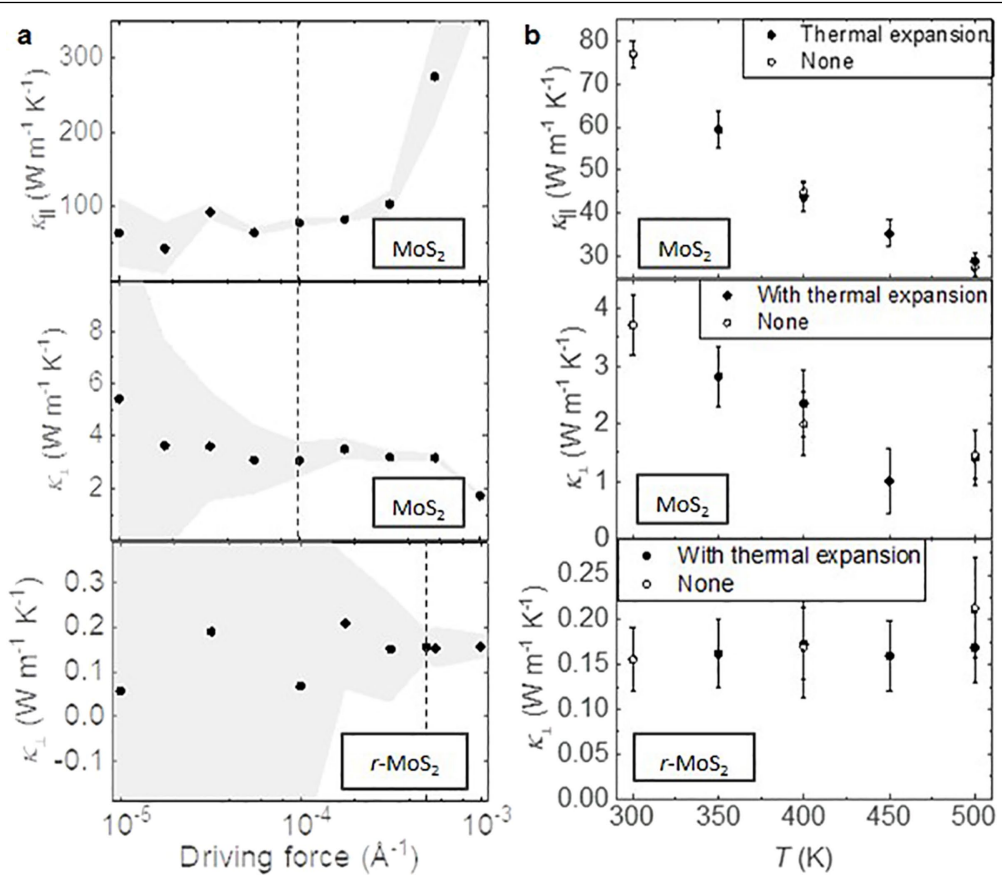

Extended Data Fig. 8 | Optimization of the MD simulations for $\boldsymbol{\kappa}$ calculations. a, Optimization of the driving force of the system, where the grey zone denotes the error. b, Effect of thermal expansion on $\kappa$. 


\section{Article}

Extended Data Table 1 | Comparison of literature thermal boundary resistances with experimentally measured values

\begin{tabular}{ll}
\hline General & $1 \mathrm{fs}$ \\
Time step & \\
\hline Equilibration & $100 \mathrm{ps}$ \\
Run time & Berendsen barostat (nvt.ber) \\
Thermostat & 0.01 \\
Thermostat coupling & \\
\hline Sampling & $1 \mathrm{~ns}$ \\
Run time & Nose-Hoover chains (nvt.nhc) \\
Thermostat & $500 \mathrm{fs}$ \\
Thermostat coupling & $1000 \mathrm{steps}$ \\
RTC interval & $1 \times 10^{-4} \mathrm{eV} / \mathrm{A} ; 5 \times 10^{-4} \mathrm{eV} / \mathrm{A}$ for $r-\mathrm{MoS}_{2}$ in the $z$ direction \\
Driving force &
\end{tabular}


Extended Data Table 2 | Parameters used in the calculations of the thermal conductivity using GPUMD

\begin{tabular}{lll}
\hline Interface & Thermal boundary resistance $\left(\mathbf{m}^{2} \mathbf{K ~ G W}-\mathbf{1}\right)$ & Reference \\
\hline Al/MoS & $\mathrm{R}_{1}$ & A. Sood et al., Nano Lett., 2019 \\
& $13-30$ & P. Jiang et al., Adv. Mater., 2017 \\
\hline $\mathrm{MoS}_{2} /$ sapphire, $\mathrm{R}_{2}$ & $20-29$ & X. F. Yue et al., J. Appl. Phys., 2020 \\
\hline $\mathrm{R}_{1}+\mathrm{R}_{2}$ & $27-53$ & \\
\hline This work (experiment) & $40-83$ & \\
& $61 \pm 7\left(r-\mathrm{MoS}_{2}\right)$ & \\
\hline
\end{tabular}

